\title{
Line profile variability from tidal interactions in binary systems
}

\author{
E. Moreno ${ }^{1}$, G. Koenigsberger ${ }^{2}$, and O. Toledano ${ }^{2}$ \\ 1 Instituto de Astronomía, Apdo. Postal 70-264, D.F. 04510, Mexico \\ e-mail: edmundo@astroscu.unam.mx \\ 2 Centro de Ciencias Físicas, UNAM, Apdo. Postal 48-3, Cuernavaca, Mor. 62251, Mexico \\ e-mail: gloria@ce.fis.unam.mx
}

Received 11 September 2004 / Accepted 5 March 2005

\begin{abstract}
We present the results of ab initio calculations of the photospheric line-profile variability produced by the tidal deformations in binary systems. We use the one-layer approximation to compute the effects produced on a primary star's equatorial region by its companion in the general case of arbitrary rotational velocity $\left(V_{\mathrm{eq}}\right)$, eccentricity and viscosity, $v$. For an eccentric binary such as $\epsilon$ Per, the computed absorption lines display bumps that are superposed on the rotationally broadened profile and that travel from the blue side of the line towards the red, qualitatively similar to the observations. The strength and multiplicity of the bumps depend on orbital phase. In the case of binaries with circular orbits, non-synchronous rotation leads to strong profile variability which can be described in terms of inverted "S"-shape patterns in grey-scale representations of the variability. The radial velocity curves obtained by measuring the centroid of the varying line-profiles are distorted with respect to the true radial velocity curves. This effect may introduce significant uncertainties in the mass determination of unseen companions. The line-profile variability is produced almost entirely by the azimuthal component of the velocity perturbations, $\Delta V_{\varphi^{\prime}}$. We put forth the hypothesis that the differentially-rotating external layers that develop in non-synchronous binary systems may lead to the generation of magnetic activity near the stellar surface. The possibility of constraining the values of $v$ near the stellar surface through the use of line-profile variability is also suggested.
\end{abstract}

Key words. stars: binaries: spectroscopic - stars: pulsation - stars: individual: $\epsilon$ Per

\section{Introduction}

Photospheric line profiles contain information on the thermal and dynamical conditions on the stellar surface. Variability of these profiles implies changing conditions in the gas layers where the absorption originates. Pulsating stars have long been known to exhibit photometric and line profile variations, and significant effort has been invested in trying to understand this variability by developing models that describe the time-dependent surface conditions of the star that may give rise to the observed variability (Osaki 1971; Smith 1977; Smith \& Buta 1979; Vogt \& Penrod 1983; Lee \& Saio 1990; Kumar et al. 1995; Telting \& Schrijvers 1997; Townsend 1997; Hao 1998; Briquet et al. 2001; Willems \& Aerts 2002; Willems 2003). The primary goal of many of these studies is to identify the pulsation modes of the stars and use this information to gain further insight into the internal stellar structure.

The current models of line-profile variability require that an assumption be made concerning the modes of pulsation and the amplitudes of the velocity variations across the stellar surface. The velocity field is described with the use of spherical harmonics, and the technique is generally limited to slow stellar rotation. In addition, pre-specified temperature variations associated with the pulsations are adopted in order to derive absorption-line profiles that resemble the observations (De Ridder et al. 2002).

In a close binary system, the problem is significantly more complicated than the case of a single star that may be oscillating with its natural frequencies. The perturbing effect of the external gravitational field may either interfere with or enhance the amplitudes of the natural frequencies, and the outcome of the interaction is strongly dependent on orbital parameters, in addition to the stellar structure and rotation. In the long term, these interactions have been shown to be a mechanism by which a binary system can reach an equilibrium state by the exchange of orbital and rotational angular momentum and energy, thus influencing the evolution of the binary system (Zahn 1970, 1977, 1989; and references therein; Press \& Teukolsky 1977; Hut 1980, 1981; Rocca 1987; Savonije et al. 1995; Goldreich \& Nicholson 1989; Smeyers et al. 1998; Witte \& Savonije 1999, 2001; Willems et al. 2001).

There is to our knowledge no model that computes at the same time the pulsation amplitudes and the expected line profiles emitted from a stellar surface that is subjected to the gravitational force of a companion. In this paper, we present a first step in 
this direction. In Sect. 2, the revised model that computes the surface oscillations (Moreno \& Koenigsberger 1999) is described; Sect. 3 presents the method for the line-profile calculation; in Sect. 4 we describe the input parameters and state the limitations of our approach; Sect. 5 provides 3 examples of the results that can be derived and Sect. 6 presents the conclusions.

\section{The surface oscillation model}

Moreno \& Koenigsberger (1999, henceforth Paper I) presented a model which allows the analysis of the surface oscillations of a star, driven by the tidal interaction with the companion in a binary system. This model calculates the solution of the equations of motion for one layer of small surface elements distributed along the equator of the star, as they respond to the forces in the system. In the non-inertial reference frame used to solve the equations of motion these forces include the gravitational forces of both stars, forces due to gas pressure, centrifugal and Coriolis forces, and viscous forces. In this model the main body of the star, interior to the thin surface layer, is assumed to behave as a rigid body, with the tidal deformation appearing mainly in the surface layer. Clearly, a detailed analysis of the general problem of oscillations induced by tidal forces requires modeling of the entire star, not the entire surface. But, in most cases, modeling the entire star requires approximations to be made, such as limiting the study to slow stellar rotational velocities and/or neglecting the presence of Coriolis and viscous forces. We are concerned primarily with the variability of the surface conditions of the star on orbital timescales and the impact that this variability may have on the photospheric absorption line profiles that are observed, regardless of the rotation rate. Furthermore, because the viscous forces act to retard or advance the location of the tidal bulge, they cannot be neglected if observed orbital phase-dependent variability is to be compared with model predictions. Hence, at this stage we have chosen to solve the problem of the line-profile variability resulting from the oscillations of the surface layer only, without imposing restrictions on the stellar rotation rate nor on the viscosity of the stellar material, while sacrificing for the moment the calculation of the response from the entire star to the tidal driving forces. Clearly, this also limits our possibilities of analysis of the free oscillation modes.

In this section we present a summary of the model, with some modifications that were introduced for the present study.

\subsection{Equations of motion}

The small surface elements whose motions are analyzed are located on the equator of one of the stars in the binary system, say star 1 with a mass $m_{1}$ and radius $R_{1}$. This equatorial plane coincides with the plane on which the motions of both stars take place, the orbital plane. The other star, star 2 with a mass $m_{2}$ and position $\boldsymbol{r}_{21}$ with respect to the center of $m_{1}$, has an instantaneous orbital angular velocity $\boldsymbol{\Omega}$ on its (in general) elliptic orbit around $m_{1}$. The motions of the surface elements in $m_{1}$ are computed in a non-inertial reference frame using Cartesian coordinates $x^{\prime}, y^{\prime}, z^{\prime}$ whose origin is at the center of $m_{1}$; the $x^{\prime}$-axis points always to star 2 and the $z^{\prime}$-axis is perpendicular to the orbital plane, its positive part giving the sense of $\boldsymbol{\Omega}$. In this reference frame the position of a surface element is $\boldsymbol{r}^{\prime}=\left(x^{\prime}, y^{\prime}, z^{\prime}\right)$. In Paper I we assigned too much detail to a surface element, considering in each element two regions in the radial direction and computing the motion of the outermost region. Here we simplify this part and compute the motion of the center of mass of each element.

Calling $\boldsymbol{a}_{\text {int }}$ the acceleration of a surface element due to gas pressure and viscous forces exerted by stellar material surrounding the element (magnetic and radiation forces are not considered), Eq. (4) in Paper I gives the total acceleration $\boldsymbol{a}^{\prime}=\left(\ddot{x}^{\prime}, \ddot{y}^{\prime}, \ddot{z}^{\prime}\right)$ of a surface element, measured in the non-inertial frame. Here we rewrite this equation as

$\boldsymbol{a}^{\prime}=\boldsymbol{a}_{\mathrm{int}}-\frac{G m_{1} \boldsymbol{r}^{\prime}}{\left|\boldsymbol{r}^{\prime}\right|^{3}}-G m_{2}\left[\frac{\boldsymbol{r}^{\prime}-\boldsymbol{r}_{21}}{\left|\boldsymbol{r}^{\prime}-\boldsymbol{r}_{21}\right|^{3}}+\frac{\boldsymbol{r}_{21}}{\left|\boldsymbol{r}_{21}\right|^{3}}\right]-\mathbf{\Omega} \times\left(\boldsymbol{\Omega} \times \boldsymbol{r}^{\prime}\right)-2 \boldsymbol{\Omega} \times \boldsymbol{v}^{\prime}-\frac{\mathrm{d} \boldsymbol{\Omega}}{\mathrm{d} t} \times \boldsymbol{r}^{\prime}$,

with $\boldsymbol{v}^{\prime}=\left(\dot{x}^{\prime}, \dot{y}^{\prime}, \dot{z}^{\prime}\right)$ the velocity of the element in the non-inertial frame. In writing the second and third terms in this equation, we ignore the effect of the tidal deformation on $m_{1}$ and $m_{2}$ on their gravitational field at the position of a surface element.

For the motion of a surface element on the stellar equator we consider a symmetry of neighboring stellar conditions in the polar direction such that $\dot{z}^{\prime}=0$. With Eq. (1), the remaining equations of motion in the $x^{\prime}$ and $y^{\prime}$ directions are

$\ddot{x}^{\prime}=\left(a_{\text {int }}\right)_{x^{\prime}}-\frac{G m_{1} x^{\prime}}{\left(x^{\prime 2}+y^{\prime 2}\right)^{3 / 2}}-G m_{2}\left[\frac{x^{\prime}-r_{21}}{\left[\left(x^{\prime}-r_{21}\right)^{2}+y^{\prime 2}\right]^{3 / 2}}+\frac{1}{r_{21}^{2}}\right]+\Omega\left(\Omega x^{\prime}+2 \dot{y}^{\prime}\right)+\dot{\Omega} y^{\prime}$,

$\ddot{y}^{\prime}=\left(a_{\text {int }}\right)_{y^{\prime}}-\frac{G m_{1} y^{\prime}}{\left(x^{\prime 2}+y^{\prime 2}\right)^{3 / 2}}-\frac{G m_{2} y^{\prime}}{\left[\left(x^{\prime}-r_{21}\right)^{2}+y^{\prime 2}\right]^{3 / 2}}+\Omega\left(\Omega y^{\prime}-2 \dot{x}^{\prime}\right)-\dot{\Omega} x^{\prime}$.

The instantaneous orbital properties $r_{21}=\left|\boldsymbol{r}_{21}\right|, \Omega=|\Omega|$, and $\dot{\Omega}$, are obtained with the two-body equations of motion

$\dot{r}_{21}=u_{r}$,

$\dot{u}_{r}=\frac{h^{2}}{r_{21}^{3}}-\frac{G\left(m_{1}+m_{2}\right)}{r_{21}^{2}}$

with $h=\Omega r_{21}^{2}=$ const., being the orbital angular momentum per unit mass. Then $\Omega=h / r_{21}^{2}, \dot{\Omega}=-2 h u_{r} / r_{21}^{3}$. 
With some hundreds for the total number of surface elements, all pairs of Eqs. (2), (3) are solved simultaneously for each element, along with the orbital Eqs. (4) and (5). We have used a seventh-order Runge-Kutta algorithm with variable step size (Fehlberg 1968) to solve these equations, but very similar results are obtained with second and fourth-order algorithms.

\subsection{Initial conditions}

In Paper I all the surface elements had the initial condition $\boldsymbol{v}^{\prime}=0$, with positions $\boldsymbol{r}^{\prime}$ taken homogeneously along a circular equatorial shell. The local initial acceleration due to surrounding material, $\boldsymbol{a}_{\text {into }}$, was forced to take a value obtained with Eq. (1) setting $\boldsymbol{a}^{\prime}=0$ and $\mathrm{d} \boldsymbol{\Omega} / \mathrm{d} t=0$, as well as $\boldsymbol{v}^{\prime}=0$. This, however, generated subsequent motions leading to a transient phase which might last several orbital periods, before a steady state solution was reached. Now we modify this scheme, taking initial conditions that shorten and smooth the transient phase. The main body of star 1 has a constant spin angular velocity $\omega_{*}$ with respect to an inertial frame. Time $t=0 \mathrm{can}$ be taken at any point of the orbit with the corresponding orbital angular velocity $\boldsymbol{\Omega}_{0}$. We define $\beta_{0}=\left|\omega_{*}\right| /\left|\boldsymbol{\Omega}_{0}\right|$ and write $\omega_{*}$ as $\omega_{*}=\beta_{0} \boldsymbol{\Omega}_{0}$. A second non-inertial reference frame is considered, with origin at the center of $m_{1}$ and rotating with the angular velocity $\omega_{*}$. Position, velocity and acceleration in this system are $\boldsymbol{r}^{\prime \prime}, \boldsymbol{v}^{\prime \prime}, \boldsymbol{a}^{\prime \prime}$. An equation similar to Eq. (1) is obtained in this reference frame changing $\boldsymbol{r}^{\prime}, \boldsymbol{v}^{\prime}, \boldsymbol{a}^{\prime}, \boldsymbol{\Omega}$ to $\boldsymbol{r}^{\prime \prime}, \boldsymbol{v}^{\prime \prime}, \boldsymbol{a}^{\prime \prime}, \boldsymbol{\omega}_{*}$ (but $\boldsymbol{r}^{\prime}=\boldsymbol{r}^{\prime \prime}$ ). At $t=0$ we take $\boldsymbol{v}^{\prime \prime}{ }_{0}=0, \boldsymbol{a}^{\prime \prime}{ }_{0}=0$ in each surface element and obtain the corresponding acceleration due to forces exerted by neighboring material

$\boldsymbol{a}_{\text {int } 0}=\left\{\frac{G m_{1} \boldsymbol{r}^{\prime}}{\left|\boldsymbol{r}^{\prime}\right|^{3}}+G m_{2}\left[\frac{\boldsymbol{r}^{\prime}-\boldsymbol{r}_{21}}{\left|\boldsymbol{r}^{\prime}-\boldsymbol{r}_{21}\right|^{3}}+\frac{\boldsymbol{r}_{21}}{\left|\boldsymbol{r}_{21}\right|^{3}}\right]\right\}_{t=0}+\beta_{0}^{2} \boldsymbol{\Omega}_{0} \times\left(\boldsymbol{\Omega}_{0} \times \boldsymbol{r}_{0}^{\prime}\right)$.

The positions $\boldsymbol{r}_{0}^{\prime}=\boldsymbol{r}^{\prime \prime}{ }_{0}$ of the surface elements are obtained with an initial spherical shape of star 1 , with radius $R_{1}$. At $t=0$ all the elements have lengths $\Delta_{0}, l_{0}$ in the radial and azimuthal directions, respectively. The initial velocity $\boldsymbol{v}_{0}^{\prime}$ is $\boldsymbol{v}_{0}^{\prime}=\left(\beta_{0}-1\right) \boldsymbol{\Omega}_{\mathbf{0}} \times \boldsymbol{r}_{\mathbf{0}}$. For a binary system with a circular relative orbit and $\beta_{0}=1$, the conditions $\boldsymbol{v}_{0}^{\prime \prime}=0, \boldsymbol{a}^{\prime \prime}{ }_{0}=0$, and $\boldsymbol{a}_{\text {int } 0}$ given by Eq. (6), are close to equilibrium conditions (i.e. $\boldsymbol{v}^{\prime \prime}=0, \boldsymbol{a}^{\prime \prime}=0$ ). In our case this equilibrium is not the initial state, due to the assumed initial spherical shape which is contrary to the tidal deformation acting on both stars.

\subsection{The acceleration $a_{\text {int }}$}

The force on a given surface element exerted by surrounding material depends on their relative motion and the dimensions of the element. We picture each element with faces pointing in the three directions of spheric coordinates, $r^{\prime}, \varphi^{\prime}, \theta^{\prime}$ in the primed non-inertial frame (the $z^{\prime}$-axis is the polar axis and $\varphi^{\prime}=0$ on the positive $x^{\prime}$-axis). At any time, the lengths $\Delta, l$ in, respectively, the radial and azimuthal directions are computed with the known positions of the centers of mass of all the elements. The length $l$ of an element is the mean of the azimuthal distances to the centers of mass of the two adjacent elements, and its length $\Delta$ is twice the distance between the center of mass of the element and the boundary of the inner, rigidly rotating, stellar region. We consider as approximately constant the length of each element in the polar direction (strictly, this is not expected to be so if the orbit is not circular). To establish the form of $\boldsymbol{a}_{\text {int }}$, we consider separately the gradient of the gas pressure and the viscous force acting on a surface element.

(1) Gradient of gas pressure.

For the interior of the elements we assume a polytropic state equation (Chandrasekhar 1957) $p=p_{0}\left(\rho / \rho_{0}\right)^{\gamma^{\prime}}, \gamma^{\prime}=1+1 / n$, with $n$ the polytropic index. We take $n=1$. Using mass conservation we have $p=p_{0}\left(l_{0} / l\right)^{\gamma^{\prime}}\left(\Delta_{0} / \Delta\right)^{\gamma^{\prime}}$. The pressure acting below an element is taken as $p_{B}=p / q$, with $0<q<1$ a constant $(q=1 / 2$ for a linear pressure gradient on the stellar surface, if we picture an underlying layer of elements in the inner stellar region, with the same local length $\Delta$ of the surface layer). With $\boldsymbol{a}_{\text {intor }}{ }^{\prime}$ the radial component of $\boldsymbol{a}_{\text {into }}$ in Eq. (6), $\delta m$ the mass of the element, $A_{0}$ the initial surface area of its faces pointing in the radial direction, and $p_{B 0}$ the local initial value of $p_{B}$, the initial outward pressure force on the element is $p_{B 0} A_{0}=p_{0} A_{0} / q=\left|\boldsymbol{a}_{\text {intor }}\right| \delta m$. Thus, in $t>0$ the outward gass pressure acceleration is

$\boldsymbol{a}_{\text {int1 }}=\frac{p_{B} A}{\delta m} \boldsymbol{e}_{r^{\prime}}=\frac{p A}{q \delta m} \boldsymbol{e}_{r^{\prime}}=\frac{p A}{p_{0} A_{0}} \boldsymbol{a}_{\mathrm{int} 0 r^{\prime}}=\left(\frac{l_{0}}{l}\right)^{\gamma^{\prime}-1}\left(\frac{\Delta_{0}}{\Delta}\right)^{\gamma^{\prime}} \boldsymbol{a}_{\mathrm{int} 0 r^{\prime}}$,

with $\boldsymbol{e}_{r^{\prime}}$ the radial unit vector.

The azimuthal gas pressure acceleration on a given element, $\boldsymbol{a}_{\mathrm{int} 2}$, is computed with the difference of the gas pressure forces exerted by the two adjacent elements. Numbering these two elements in the direction of azimuth increase, and with $S$ the surface area of the faces of the element pointing in the azimuthal direction, $\boldsymbol{a}_{\text {int2 }}$ is given by $\boldsymbol{a}_{\text {int2 }}=\left(p_{1}-p_{2}\right) S \boldsymbol{e}_{\varphi^{\prime}} / \delta m=q\left(p_{1}-\right.$ $\left.p_{2}\right) S\left|\boldsymbol{a}_{\text {int } 0 r^{\prime}}\right| \boldsymbol{e}_{\varphi^{\prime}} /\left(p_{0} A_{0}\right)$, with $\boldsymbol{e}_{\varphi^{\prime}}$ the azimuthal unit vector. Introducing the expressions of the adjacent polytropic pressures $p_{1}, p_{2}$ and approximating their initial values as the initial pressure $p_{0}$ corresponding to the given (central) element, $\boldsymbol{a}_{\text {int2 }}$ can be written as

$\boldsymbol{a}_{\mathrm{int2}}=q\left(\frac{\Delta}{l_{0}}\right)\left(l_{0} \Delta_{0}\right)^{\gamma^{\prime}}\left\{\left(l_{1} \Delta_{1}\right)^{-\gamma^{\prime}}-\left(l_{2} \Delta_{2}\right)^{-\gamma^{\prime}}\right\}\left|\boldsymbol{a}_{\mathrm{int} 0 r^{\prime}}\right| \boldsymbol{e}_{\varphi^{\prime}}$. 
(2) Viscous force.

Now we consider the shear part of the kinematic stress tensor of the stellar material. This part is given by (Symon 1971):

$\boldsymbol{P}_{\eta}=-\eta\left[\nabla^{\prime} \boldsymbol{v}^{\prime}+\left(\nabla^{\prime} \boldsymbol{v}^{\prime}\right)^{t r p}-\frac{2}{3} \mathbf{1}\left(\nabla^{\prime} \cdot \boldsymbol{v}^{\prime}\right)\right]$

with 1 the unit matrix, trp indicating the transposed, and $\eta$ the coefficient of dynamic viscosity, related to the coefficient of kinematic viscosity, $v$, by $\eta=v \rho$ (Landau \& Lifshitz 1984), with $\rho$ the mass density. The total force on a given element due to shear stresses is $\boldsymbol{F}_{\eta}=-\oint \boldsymbol{P}_{\eta} \cdot \mathrm{d} \boldsymbol{S}$, with the integration over its surface. The outer face in the radial direction does not contribute to $\boldsymbol{F}_{\eta}$, and also we assume that the contribution is small on the two faces in the polar direction. Doing the integration on the remaining faces in the azimuthal and radial directions, and ignoring expected small terms, $\boldsymbol{F}_{\eta}$ is approximately

$\boldsymbol{F}_{\eta} \simeq \eta S\left\{\left(\frac{1}{r^{\prime}} \frac{\partial v_{r^{\prime}}^{\prime}}{\partial \varphi^{\prime}}\right)_{2}-\left(\frac{1}{r^{\prime}} \frac{\partial v_{r^{\prime}}^{\prime}}{\partial \varphi^{\prime}}\right)_{1}\right\} \boldsymbol{e}_{r^{\prime}}-\eta A\left(\frac{\partial v_{\varphi^{\prime}}^{\prime}}{\partial r^{\prime}}-\frac{v_{\varphi^{\prime}}^{\prime}}{r^{\prime}}\right)_{\mathrm{A}} \boldsymbol{e}_{\varphi^{\prime}}$.

The first term in Eq. (10) comes from the integration on the azimuthal faces, with surface area $S$, and the second term is the main contribution on the lower radial face with surface area $A$.

With $\delta m=\rho S l, \eta S=v \rho S,\left(v_{r^{\prime}}^{\prime}\right)_{1},\left(v_{r^{\prime}}^{\prime}\right)_{2},\left(v_{r^{\prime}}^{\prime}\right)_{c}$ the radial velocities of the two adjacent elements and the given central element, respectively, and approximating $\left(\partial v_{r^{\prime}}^{\prime} / r^{\prime} \partial \varphi^{\prime}\right)_{1} \simeq\left[\left(v_{r^{\prime}}^{\prime}\right)_{c}-\left(v_{r^{\prime}}^{\prime}\right)_{1}\right] / l,\left(\partial v_{r^{\prime}}^{\prime} / r^{\prime} \partial \varphi^{\prime}\right)_{2} \simeq\left[\left(v_{r^{\prime}}^{\prime}\right)_{2}-\left(v_{r^{\prime}}^{\prime}\right)_{c}\right] / l$, the radial acceleration due to shear stresses, $\boldsymbol{a}_{\text {int3 }}=\left(\boldsymbol{F}_{\eta} \cdot \boldsymbol{e}_{r^{\prime}}\right) \boldsymbol{e}_{r^{\prime}} / \delta m$, is

$\boldsymbol{a}_{\mathrm{int} 3} \simeq \frac{v}{l^{2}}\left[\left(v_{r^{\prime}}^{\prime}\right)_{2}+\left(v_{r^{\prime}}^{\prime}\right)_{1}-2\left(v_{r^{\prime}}^{\prime}\right)_{c}\right] \boldsymbol{e}_{r^{\prime}}$

The azimuthal acceleration due to shear stresses, $\boldsymbol{a}_{\text {aint4 }}=\left(\boldsymbol{F}_{\eta} \cdot \boldsymbol{e}_{\varphi^{\prime}}\right) \boldsymbol{e}_{\varphi^{\prime}} / \delta m$, can be written as $\boldsymbol{a}_{\text {int4 }}=-(\eta A / \delta m)\left(r^{\prime} \partial \omega^{\prime} / \partial r^{\prime}\right)_{\mathrm{A}} \boldsymbol{e}_{\varphi^{\prime}}$, with $\omega^{\prime}=v_{\varphi^{\prime}}^{\prime} / r^{\prime}$ angular velocity computed in the primed non-inertial frame. We approximate $\left(\partial \omega^{\prime} / \partial r^{\prime}\right)_{\mathrm{A}}$ as $\left(\partial \omega^{\prime} / \partial r^{\prime}\right)_{\mathrm{A}} \simeq\left(\omega^{\prime}-\omega_{\star}^{\prime}\right) / \Delta$, with $\omega^{\prime}$ the angular velocity of the element and $\omega_{\star}^{\prime}=\beta_{0}\left|\Omega_{0}\right|-|\boldsymbol{\Omega}|$ the angular velocity of the rigidly rotating stellar region, measured in the primed non-inertial frame. Also, we approximate $r_{\mathrm{A}}^{\prime} \simeq r^{\prime}$, with $r^{\prime}$ the radial position of the element. Then, with $\delta m=\rho A \Delta, \eta A=v \rho A$, and $v_{\varphi^{\prime}}^{\prime}$ being the azimuthal velocity of the element, the acceleration $\boldsymbol{a}_{\text {int } 4}$ is

$\boldsymbol{a}_{\mathrm{int} 4} \simeq-\frac{v}{\Delta^{2}}\left[v_{\varphi^{\prime}}^{\prime}-r^{\prime}\left(\beta_{0}\left|\boldsymbol{\Omega}_{0}\right|-|\boldsymbol{\Omega}|\right)\right] \boldsymbol{e}_{\varphi^{\prime}}$

The total acceleration $\boldsymbol{a}_{\text {int }}$ is the sum of the accelerations given in Eqs. (7), (8), (11) and (12).

\section{Model computation of a line profile}

In this section we give the procedure to compute an absorption line profile with the stellar surface motions resulting from applying the model in Sect. 2. As we are only computing the motion of a surface layer on the stellar equator, we need first to extend the resulting motion and deformation to other latitudes $\theta^{\prime}$. The surface profile $r^{\prime}\left(\varphi^{\prime}\right)$ obtained on the stellar equator is extended to latitude $\theta^{\prime}, r_{\mathrm{ex}}^{\prime}\left(\varphi^{\prime}, \theta^{\prime}\right)$, by means of the relation

$r_{\mathrm{ex}}^{\prime}\left(\varphi^{\prime}, \theta^{\prime}\right)=R_{1}+\left(r^{\prime}\left(\varphi^{\prime}\right)-R_{1}\right) \sin \theta^{\prime}$

In the following it is supposed that $\left|r^{\prime}-R_{1}\right| / R_{1}$ is small, thus relation (13) is a minor extension.

With the velocities $v_{r^{\prime}}^{\prime}, v_{\varphi^{\prime}}^{\prime}$ that result from the calculation on the stellar equator, the extension to latitude $\theta^{\prime}$ is obtained using the relations

$\left(v_{r^{\prime}}^{\prime}\right)_{\mathrm{ex}}=v_{r^{\prime}}^{\prime} \sin \theta^{\prime}$

$\left(v_{\varphi^{\prime}}^{\prime}\right)_{\mathrm{ex}}=v_{\varphi^{\prime}}^{\prime} \frac{r_{e x}^{\prime}\left(\varphi^{\prime}, \theta^{\prime}\right)}{r^{\prime}\left(\varphi^{\prime}\right)} \sin \theta^{\prime}$,

and zero velocity is considered in the $\theta^{\prime}$ direction. For a given azimuth $\varphi^{\prime}$, relation (15) gives the same rotational angular velocity at every polar angle $\theta^{\prime}$.

An inertial reference frame is now considered in which the center of mass of the binary system is at rest. For an observer in this frame, far away from the binary system, a surface element in star 1 has spherical azimuth and polar angles $(\varphi, \theta)$, with $\theta$ measured from the line connecting the observer and the stellar center and being the angle between the line-of-sight and the normal to the stellar surface at the position of the element. The azimuth $\varphi$ is measured from an arbitrary line on the stellar image, as seen by the observer.

In the local reference frame of a surface element, an absorption line with center at a wavelength $\lambda_{0}$ has an intrinsic shape $f(\zeta)$ assumed independent of the position of the element, and depending on wavelength through the variable $\zeta=\left(\lambda_{0}-\lambda\right) / \lambda_{0}$. Also, $s(\theta)$ is the limb darkening factor and $a(\varphi, \theta)$ is the line-contrast factor. 


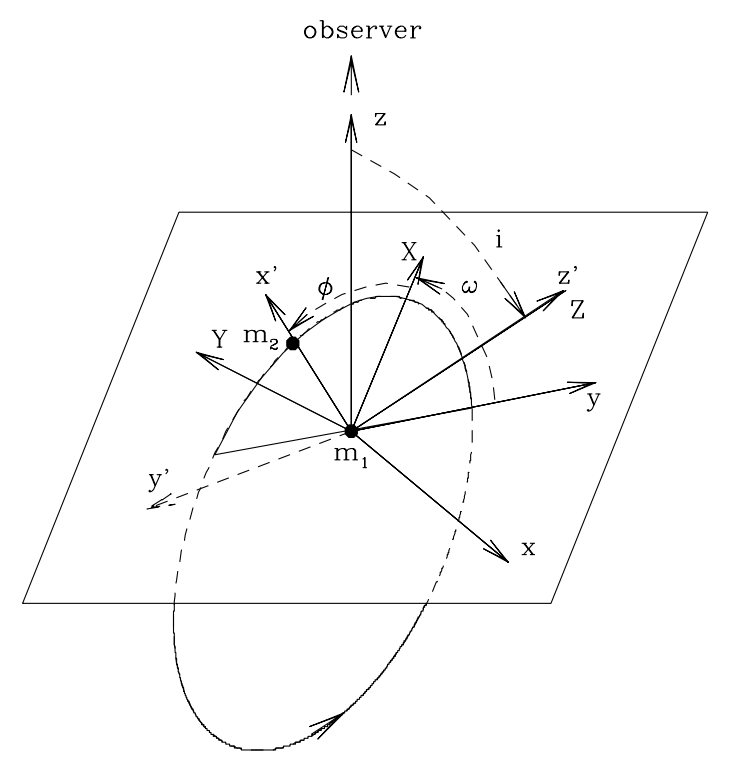

Fig. 1. The three Cartesian systems, centered on star 1 , used to obtain the transformation $(\varphi, \theta) \leftrightarrow\left(\varphi^{\prime}, \theta^{\prime}\right)$.

Following the fundamental discussion given by Carroll (1928), the absorption line profile seen by the observer is

$h_{\mathrm{obs}}\left(\zeta^{\star}\right)=1-\frac{\int_{0}^{2 \pi} \mathrm{d} \varphi \int_{0}^{\pi / 2} s(\theta) a(\varphi, \theta) f\left(\zeta^{\star}+\frac{\left(v_{r}\right)_{\mathrm{obs}}(\varphi, \theta)}{c}\right) \sin \theta \cos \theta \mathrm{d} \theta}{2 \pi \int_{0}^{1} s[\mu] \mu \mathrm{d} \mu}$,

with $\zeta^{\star}=\left(\lambda_{0}-\lambda^{\prime}\right) / \lambda_{0}, \lambda^{\prime}$ the wavelength in the observer's frame, $\left(v_{r}\right)_{\text {obs }}(\varphi, \theta)$ the radial velocity with respect to the observer of a visible surface element in star 1 , the coordinates of the element being $(\varphi, \theta), c$ the speed of light, $\mu=\cos \theta$, and $s[\mu]=s(\arccos \mu)$. In our computations we consider $a(\varphi, \theta)=a_{0}$, a constant, $s(\theta)=(1-u+u \cos \theta)$, where we choose the limb-darkening coefficient $u=0.6$ (in agreement with the Milne-Eddington approximation), and a Gaussian shape function $f(\zeta)=\mathrm{e}^{-\left(c / v_{t}\right)^{2} \zeta^{2}}$, where $v_{\mathrm{t}}$ is the effective thermal velocity and $c$ is the speed of light.

The double integration in Eq. (16) is over the surface of star 1 visible to the observer. Given a point with coordinates $(\varphi, \theta)$ in the range of integration (i.e., in the visible stellar disk) we need its corresponding coordinates $\left(\varphi^{\prime}, \theta^{\prime}\right)$ (see Sect. 2.3) to identify the surface element. Thus, we know $\boldsymbol{v}^{\prime}$ at this point and $\left(v_{r}\right)_{\mathrm{obs}}$ can be computed and introduced in Eq. (16).

In Fig. 1 we show three Cartesian systems, centered on star 1 , which help to obtain the transformation $(\varphi, \theta) \leftrightarrow\left(\varphi^{\prime}, \theta^{\prime}\right)$. The axes $x, y$ lie on the plane of the sky for the observer, with the positive $y$-axis pointing to the ascending node of the orbit of $m_{2}$ around $m_{1}$; the positive $z$-axis points to the observer. The spherical angles $\varphi, \theta$ are measured in these Cartesian axes $x, y, z: \varphi=0$ on the positive $x$-axis and $\theta=0$ on the positive $z$-axis. Also shown in Fig. 1 are the Cartesian axes $x^{\prime}, y^{\prime}, z^{\prime}$ introduced in Sect. 2: the axes $x^{\prime}, y^{\prime}$ lie on the orbital plane, with the positive $x^{\prime}$-axis pointing to star 2 ; the positive $z^{\prime}$-axis points in the sense of the orbital angular velocity $\Omega$. The spherical angles $\varphi^{\prime}, \theta^{\prime}$ are measured in this Cartesian system (see Sect. 2.3). The third Cartesian system in Fig. 1 is shown with axes $X, Y, Z$. The $Z$-axis is the same as the $z^{\prime}$-axis and the positive $X$-axis points to periastron. We give the inclination $i$ of the orbit, the argument of periastron, $\omega$, and the angular position $\phi$ of $m_{2}$ with respect to the periastron line ( $\phi$ is the true anomaly).

With the transformations $(x, y, z) \leftrightarrow(X, Y, Z),(X, Y, Z) \leftrightarrow\left(x^{\prime}, y^{\prime}, z^{\prime}\right)$, and writing the Cartesian coordinates $(x, y, z),\left(x^{\prime}, y^{\prime}, z^{\prime}\right)$ in corresponding spherical coordinates, we obtain

$$
\left(\begin{array}{c}
\sin \theta \cos \varphi \\
\sin \theta \sin \varphi \\
\cos \theta
\end{array}\right)=\left(\begin{array}{ccc}
-\sin (\omega+\phi) \cos i & -\cos (\omega+\phi) \cos i & \sin i \\
\cos (\omega+\phi) & -\sin (\omega+\phi) & 0 \\
\sin (\omega+\phi) \sin i & \cos (\omega+\phi) \sin i & \cos i
\end{array}\right)\left(\begin{array}{c}
\sin \theta^{\prime} \cos \varphi^{\prime} \\
\sin \theta^{\prime} \sin \varphi^{\prime} \\
\cos \theta^{\prime}
\end{array}\right)
$$

Taking the inverse of the transformation in Eq. (17), the angles $\left(\varphi^{\prime}, \theta^{\prime}\right)$ are computed for the corresponding pair $(\varphi, \theta)$ on the visible stellar disk.

To obtain $\left(v_{r}\right)_{\text {obs }}$, we know that the velocity of a surface element in $m_{1}$ with respect to the inertial frame in the center of mass of the binary system is $\boldsymbol{v}_{\mathrm{el}}=\boldsymbol{v}_{1}+\boldsymbol{v}^{\prime}+\boldsymbol{\Omega} \times \boldsymbol{r}^{\prime}$, with $\boldsymbol{v}_{1}$ the velocity of $m_{1}$ with respect to the center of mass and $\boldsymbol{r}^{\prime}, \boldsymbol{v}^{\prime}$ the position and velocity of the element in the primed non-inertial frame introduced in Sect. 2. Also, the position of $m_{2}$ with respect to $m_{1}$ 
is $\boldsymbol{r}_{21}=-\left(1+\frac{m_{1}}{m_{2}}\right) \boldsymbol{r}_{1}$, with $\boldsymbol{r}_{1}$ the position of $m_{1}$ with respect to the center of mass. Using this relation, the velocity of $m_{2}$ in the $(X, Y, Z)$ frame shown in Fig. 1 is $\boldsymbol{V}_{2}=-\left(1+\frac{m_{1}}{m_{2}}\right) \boldsymbol{v}_{1}$. Thus, $\left(v_{r}\right)_{\mathrm{obs}}$ is obtained with

$\left(v_{r}\right)_{\mathrm{obs}}=-\boldsymbol{v}_{\mathrm{el}} \cdot \boldsymbol{k}=-\left(-\frac{m_{2}}{m_{1}+m_{2}} \boldsymbol{V}_{2}+\boldsymbol{v}^{\prime}+\boldsymbol{\Omega} \times \boldsymbol{r}^{\prime}\right) \cdot \boldsymbol{k}$,

with $\boldsymbol{k}$ the unit vector along the $z$-axis.

In terms of the orbital eccentricity, $e$, the major semi-axis, $a$, the mean motion $n=\sqrt{G\left(m_{1}+m_{2}\right)} / a^{3 / 2}$ and the eccentric anomaly $E$, with $\cos E=\left(a-r_{21}\right) / a e$, the velocity $\boldsymbol{V}_{2}$ is

$$
\boldsymbol{V}_{2}=-\frac{n a \sin E}{1-e \cos E} \boldsymbol{i}_{X}+\frac{n a \sqrt{1-e^{2}} \cos E}{1-e \cos E} j_{Y}
$$

with $\boldsymbol{i}_{X}, \boldsymbol{j}_{Y}$ the unit vectors along the $X$ and $Y$ axes, respectively.

The remaining term $\boldsymbol{v}^{\prime}+\boldsymbol{\Omega} \times \boldsymbol{r}^{\prime}$ in $\boldsymbol{v}_{\mathrm{el}}$ can also easily be written in the $(X, Y, Z)$ frame. Thus, with $\boldsymbol{k}=\boldsymbol{i}_{X} \sin i \sin \omega+$ $\boldsymbol{j}_{Y} \sin i \cos \omega+\boldsymbol{k}_{Z} \cos i,\left(v_{r}\right)_{\mathrm{obs}}$ in Eq. (18) is given by

$$
\begin{aligned}
\left(v_{r}\right)_{\mathrm{obs}}= & -\left\{\frac{m_{2} n a \sin E}{\left(m_{1}+m_{2}\right)(1-e \cos E)}+\dot{x}^{\prime} \cos \phi-\dot{y}^{\prime} \sin \phi-\Omega\left(x^{\prime} \sin \phi+y^{\prime} \cos \phi\right)\right\} \sin i \sin \omega \\
& -\left\{-\frac{m_{2} n a \sqrt{1-e^{2}} \cos E}{\left(m_{1}+m_{2}\right)(1-e \cos E)}+\dot{x}^{\prime} \sin \phi+\dot{y}^{\prime} \cos \phi+\Omega\left(x^{\prime} \cos \phi-y^{\prime} \sin \phi\right)\right\} \sin i \cos \omega \\
& -\dot{z}^{\prime} \cos i
\end{aligned}
$$

with $\boldsymbol{r}^{\prime}$ and $\boldsymbol{v}^{\prime}$ corresponding to the surface element with coordinates $\left(\varphi^{\prime}, \theta^{\prime}\right)$.

\section{Description of the parameters and limitations of the model}

\subsection{Stellar, orbital and rotation parameters}

In order to compute the line profiles, the following parameters that describe the stars and the binary system are required: the stellar masses $\left(m_{1}, m_{2}\right)$, the orbital period $\left(P_{\text {orb }}\right)$ and eccentricity $(e)$, the inclination of the orbital plane $(i)$, the argument of periastron $(\omega)$, the radius of star $m_{1}\left(R_{1}\right)$ and the stellar rotation parameter, $\beta_{0}=\left|\omega_{*}\right| /\left|\mathbf{\Omega}_{0}\right|$. This parameter requires knowledge of the equatorial rotational velocity, $V_{\mathrm{eq}} \simeq R_{1}\left|\omega_{*}\right|$. It is important to note that in the case of eccentric orbits, the rotation parameter is not constant throughout the orbital cycle because the orbital angular velocity, $\left|\boldsymbol{\Omega}_{\mathbf{0}}\right|$, is a function of position on the orbit. We characterize the rotation properties of the eccentric binary systems with the value of the rotation parameter at periastron, $\beta_{\text {per }}$. Hence, for example, if $\beta_{\text {per }}=1$, the system is in synchronous rotation at periastron, but it is super-synchronous at all other orbital phases.

A second point to note is the fact that in any non-synchronously rotating star, the rotation parameter is not constant over the stellar surface. For example, the fluid which is near the limb that is rotating in the direction of $m_{2}$ responds to the gravitational pull of $m_{2}$ by accelerating slightly, while the motion of the fluid near the opposite limb is slightly retarded. Hence, it is to be understood that the value of $\beta_{0}$ or $\beta_{\text {per }}$ in an eccentric orbit that we cite corresponds to the rotation rate of the underlying body of the star, that is assumed to rotate as a rigid body. The actual angular velocity of each surface element is one of the products of our calculation.

\subsection{Kinematic viscosity}

The kinematic viscosity, $v$, is the most difficult parameter to establish for our calculations because, in general, its values are not known. This parameter significantly influences the amplitudes of the oscillations; if, for example, it is very large, the resulting amplitudes are too small. On the other hand, if it is too small, the oscillation amplitudes of individual surface elements may lead them to overlap with neighboring elements. This condition prevents the numerical calculation from proceding, and imposes a lower limit for the value of $v$ in our numerical method. We have chosen values of $v$ for our calculations as close as possible to this minimum, which, for the type of binary systems analyzed in this paper are typically in the range $0.002-0.1 R_{\odot}^{2}$ day $^{-1}$.

The viscosity also plays an important role in the studies of accretion disks through the $\alpha$-parameter (Shakura \& Sunyaev 1973) which, neglecting magnetic fields, is related to the kinematic viscosity through $\eta \omega_{*}=\alpha P$, where $P$ is the pressure and $\eta=\rho v$, with $\rho$ the mass density. Thus, writing $v=\frac{k T}{\mu m_{H}} \frac{\alpha}{\omega_{*}}$, and assuming $T=30000 \mathrm{~K}$ and $\omega_{*}=3 \times 10^{-5} \mathrm{~s}^{-1}$, the range of $v$ values given above corresponds to a range in the $\alpha$ parameter $0.001-0.5$. 


\subsection{The surface layer}

Because we solve the equations of motion of the surface layer only, the thickness chosen for this layer is important. Typically, the surface layer has a thickness in the range $0.005-0.01 R_{1}$.

The number of segments, $N_{\text {seg }}$ into which the surface layer is divided determines the resolution in the azimuthal $(\varphi)$ direction for calculation of the oscillations. We generally divide the equatorial belt into 500 elements. For the line-profile calculation, $N_{\theta}$ and $N_{\varphi}$ define the number of locations across the visible stellar surface at which the line-of-sight velocity components are to be provided. We generally use $N_{\theta}=N_{\varphi}=500$.

\subsection{Line-profile parameters}

The two parameters used to define the line profile are $a_{0}$, the central depth of the line on a normalized continuum spectrum, and $c / v_{\mathrm{t}}$, which determines the width of the Gaussian profile. Here, $v_{\mathrm{t}}$ may be associated with the thermal or the microturbulent speeds. Model fitting to spectral lines of hot stars suggests large values of $v_{\mathrm{t}}$, sometimes corresponding to velocities near the sound speed (Lennon et al. 1991; Hubeny et al. 1991). We use $v_{\mathrm{t}}=10 \mathrm{~km} \mathrm{~s}^{-1}$ in the examples that will be presented in the following sections. With larger values of $v_{\mathrm{t}}$, the local broadening leads to blending of contiguous narrow features, and therefore fewer narrow bumps are resolved within the line profile. Note that the computation of line profile variations based on local intensity profiles taken to be Gaussians is not correct, because the actual local intensity profiles tend to be saturated and box-shaped (M. Smith, private communication), thus enhancing the actual amplitude of variability with respect to that produced with Gaussian profiles. Hence, our calculations should underpredict the variability amplitudes.

\subsection{Summary of the model limitations}

The response of the star to the external gravitational potential is assumed to be limited to a surface layer along the equatorial region. Thus, the resulting free oscillations only reflect the behavior of this single layer while, in reality, the oscillations are a characteristic of the entire stellar structure.

The calculations are performed only for the equatorial belt. For the line-profile calculation, the results obtained for the equator are extended to other latitudes assuming that the amplitudes obey a monotonically decreasing function of latitude. Thus, although the Coriolis force is included in the computations, the circulation currents that they could produce in a 3-D calculation are absent in the present calculations. Such circulation patterns could be associated with toroidal modes suggested to be present in the binary system $\alpha$ Vir by Smith (1985a,b). In addition, we assume that the stellar rotation axis is perpendicular to the orbital plane, which is not necessarily the case in general.

The oscillations that are excited by the tidal interaction are assumed to be adiabatic; thus, radiative damping is neglected, as are the potential effects of stellar winds. In addition, the possible temperature variations associated with the oscillations are neglected. This is justified, at least for the case of the B0.5 IV star $\epsilon$ Per that we consider below, because its line profile variability is found to be dominated by the oscillatory velocity field, rather than the oscillatory temperature variations (Telting $\&$ Schrijvers 1997).

We assume a constant value of the kinematic viscosity, $v$. However, there is no reason a priori to expect that $v$ should remain constant under the varying conditions of the stellar surface. In particular, the magnitude of $v$ is expected to increase significantly if there are regions near the stellar surface with strong magnetic fields.

\section{Examples}

In this section we describe the results of the calculations for three test cases: 1) an equilibrium configuration; 2) an eccentric orbit; and 3) a circular orbit with non-synchronous rotation. The input parameters for these three cases are listed in Table 1. We will show that the profile variations that result from our calculations display excess absorption and excess emission (i.e., bumps) superposed on the rotationally-broadened profile and that travel across the line profile, starting near the blue edge and disappearing near the red edge. This behavior is observed in numerous variable stars.

\subsection{System in equilibrium}

A binary system can be in equilibrium when the following three conditions are satisfied: 1) the orbit is circular; 2) the stellar rotation period equals the orbital period (i.e., corotation); and 3) the equators of both stars lie in the orbital plane. As a test case for the equilibrium configuration, we have chosen a binary system with relatively massive components $\left(17.8\right.$ and $\left.10.1 M_{\odot}\right)$, in a $P_{\text {orb }}=56$ day orbit.

In order to represent the temporal behavior of the surface oscillations, we use the quantities $R_{\max }$ and $V_{\max }$, as illustrated in Fig. 2. $R_{\max }$ (top panel) is the maximum radius of the star, generally associated with the primary tidal bulge. Likewise, $V_{\max }$ is the velocity component in the radial direction of the surface element which is expanding fastest. $R_{\max }$ and $V_{\max }$ are not associated 
Table 1. Parameters for test cases.

\begin{tabular}{|c|c|c|c|}
\hline Parameter & Equilibrium & Eccentric $(\epsilon$ Per $)$ & Circular \\
\hline$m_{1}\left(M_{\odot}\right)$ & 17.8 & 13.5 & 13.5 \\
\hline$m_{2}\left(M_{\odot}\right)$ & 10.1 & 2.0 & 2.0 \\
\hline$P_{\text {orb }}$ (days) & 56.0 & 14.08 & 4.03 \\
\hline$e$ & 0. & 0.559 & 0. \\
\hline$i\left(^{\circ}\right)$ & 90 & 90 & 90 \\
\hline$\omega\left(^{\circ}\right)$ & - & 110 & - \\
\hline \multirow[t]{2}{*}{$\beta_{0}$} & 1.00 & - & $1.50,1.0$ \\
\hline & & & $0.77,0.60$ \\
\hline$\beta_{\text {per }}$ & - & 1.50 & - \\
\hline$R_{1}$ & 16.25 & 6.90 & 6.90 \\
\hline$v\left(R_{\odot}^{2} \mathrm{day}^{-1}\right)$ & 0.001 & 0.002 & 0.010 \\
\hline$\delta R / R_{1}$ & 0.005 & 0.010 & 0.010 \\
\hline$N_{\text {seg }}$ & 500 & 500 & 500 \\
\hline$N_{\theta}, N_{\varphi}$ & 500,500 & 500,500 & 500,500 \\
\hline$a_{0}$ & 0.7 & 0.9 & 0.9 \\
\hline$c / v_{\mathrm{t}}$ & 8000 & 30000 & 30000 \\
\hline
\end{tabular}

with the same surface element. When the calculation is started, the initially spherical shape of the star $m_{1}$ immediately responds to the gravitational field of the companion and is distorted. This is followed by a number of oscillations with decreasing amplitudes until a stable configuration is achieved. One thousand days after the start of the calculations, $\Delta R_{\max } / R_{1}<10^{-8}$. The equilibrium value of $R_{\max }\left(16.2513 R_{\odot}\right)$ corresponds to the radius of the star at the location of the tidal bulge. The orientation of the tidal bulge deviates by $+0.01^{\circ}$ from the line connecting both stars; i.e., it is almost precisely aligned with the direction of the companion. The variations in $V_{\max }$ also die down on the timescale of one orbital cycle, so that by $t \sim 1000$ days, $V_{\max }<10^{-4} \mathrm{~km} \mathrm{~s}^{-1}$. No line-profile variability is produced.

The value of $R_{\max }$ obtained above is smaller than the maximum deformation predicted by the classical Roche model using (Chandrasekhar 1933):

$\frac{\mathrm{d} x}{R_{1}}=\left(\frac{R_{1}}{D}\right)^{3}\left[\frac{1}{6}\left(1+7 \frac{m_{2}}{m_{1}}\right) \delta+2\left(1+\frac{m_{2}}{m_{1}}\right) f\right]$

where $D$ is the separation of the two stars' centers and $\delta$ and $f$ are parameters that depend on the value of the polytropic index. Adopting the polytropic index $n=1$, then $\delta=1.52$ and $f=0.1$. Inserting the values for the equilibrium binary system we obtain $\mathrm{d} x / R_{1}=0.00116$ and hence, $R_{\max }^{\text {Roche }}=16.269$. However, in our model the centers of mass of the surface elements do not necessarily lie on an equipotential surface and, therefore, a direct comparison with the classical Roche model is not very meaningful.

\subsection{Eccentric orbit}

\subsubsection{Predicted line profile variations in $\epsilon$ Per}

To illustrate the effects of a moderately eccentric orbit, we have chosen the parameters of the highly variable binary system $\epsilon$ Per. This system has been extensively studied, and examples of its line profile variability are available in the literature (Smith et al. 1987; Gies \& Kullavanijaya 1988; Harmanec 1989; Harmanec \& Tarasov 1990; Tarasov et al. 1995; Telting \& Schrijvers 1997; Gies et al. 1999; De Cat et al. 2000). The first discovery that blue-to-red moving bumps on the photospheric absorptions produce the striking line profile variability in this system was made by Bolton (1983). Smith et al. (1987) attributed this variability to photospheric nonradial pulsations (NRPs), a conclusion that was supported by Gies \& Kullavanijaya (1988). However, Harmanec (1989, 1999) proposed that an alternative mechanism causing the line profile variability could be the presence of corotating structures located above the photosphere. Gies et al. (1999) show that the blue-to-red moving bumps are also present in UV photospheric lines, and that their motion is correlated with that of optical lines. They reach the conclusion that only NRPs can explain the variability. Saio et al. (2000) also conclude that the variations observed by Gies et al. (1999) can be modeled with NRPs. They also find that the periods in the range 2-4 h are consistent with the Z-bump kappa mechanism (Kiriakidis et al. 1992; Moskalik \& Dziembowski 1992), although they are unable to explain all the characteristics of the variability with this driving mechanism. 

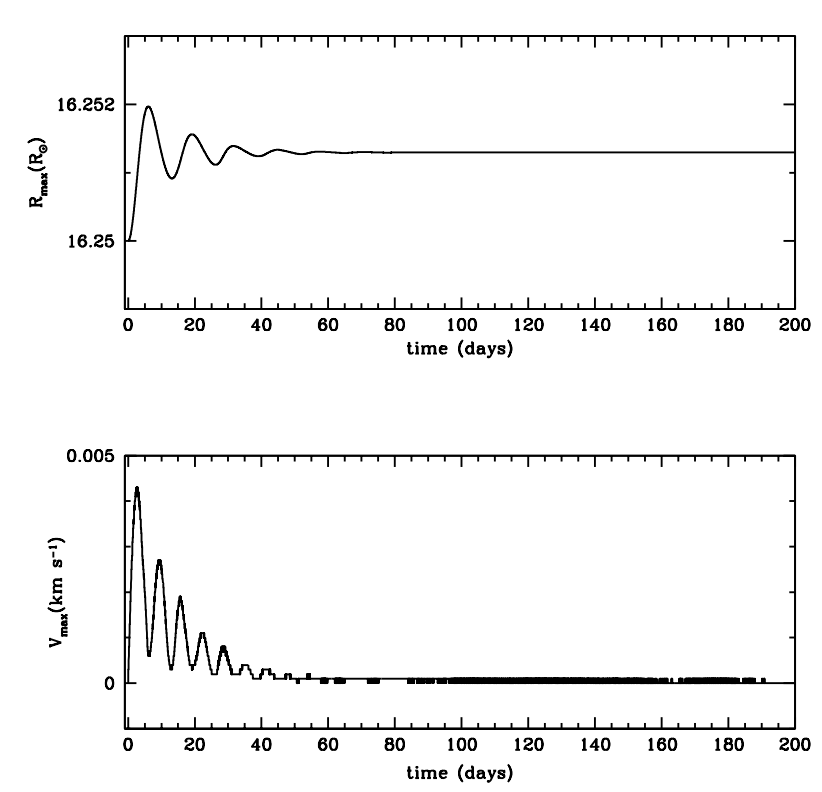

Fig. 2
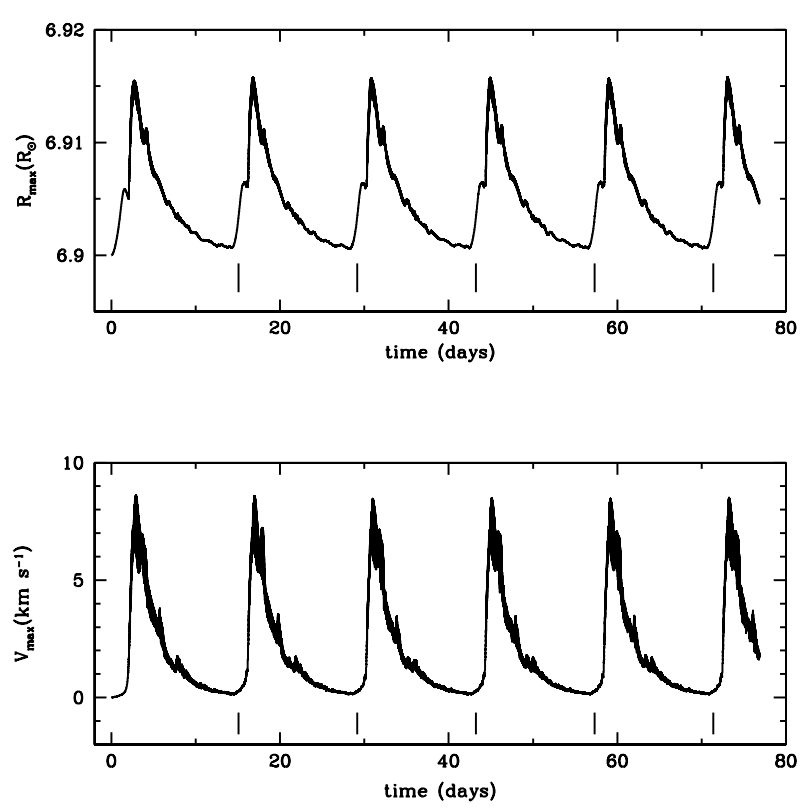

Fig. 3

Fig. 2. Time-dependent behavior of the maximum radius (top) and radial velocity (bottom) of the surface elements on a $17.8+10.1 M_{\odot}$ binary system with a $P=56$ days circular orbit. The system is in synchronous rotation. After the initial transitory phase that lasts approximately one orbital cycle, nearly no fluctuations in $R_{\max }$ are present: at $t \sim 1000$ days, $\Delta R_{\max } / R_{1}<10^{-8}$. The corresponding variations in $V_{\max }<10^{-4} \mathrm{~km} \mathrm{~s}^{-1}$, thus showing that the code reaches the equilibrium configuration relatively quickly and does not deviate from this solution as time progresses.

Fig. 3. Temporal behavior of $R_{\max }$ and $V_{\max }$ for a model computed with input parameters corresponding to those of $\epsilon$ Per and $v=0.002 R_{\odot}^{2}$ day ${ }^{-1}$. The large tick marks indicate times of periastron passage.

In Table 1 we list the parameters that we used to perform the model calculation, and that were taken from the data listed by Tarasov et al. (1995) and De Cat et al. (2000). Figure 3 illustrates the temporal behavior of $R_{\max }$ and $V_{\max }$ as computed with our model. Periastron passage is indicated with the large tick marks, showing that maximum deformation and radial velocity occur $\sim 2$ days after periastron. In addition to the large scale variations that occur on orbital timescales, Fig. 3 indicates the presence of variability on shorter timescales. Oscillations are clearly seen with $P_{\mathrm{osc}} \sim 16$ min that can be associated with the natural oscillation frequency of the surface layer. In addition to these, a modulation with longer timescales is also present.

Each cycle presents nearly identical characteristics. We selected the cycle that starts with periastron passage on day 57.3 of the calculation, and computed line profiles separated in time by 0.02 day covering the entire orbital cycle. A sample of these profiles is illustrated in Fig. 4, compared with the corresponding rotationally-broadened, but unperturbed line profiles. A variety of shapes are obtained, some with multiple bumps and several wiggles, with a clear tendency towards numerous weak and narrow bumps near apastron and fewer but stronger and broader bumps around periastron. The strongest perturbation of the line profiles occurs very near periastron, when the forcing due to star $m_{2}$ 's proximity is greatest.

Figure 5 illustrates a sequence of profiles separated in time by 0.1 day starting on day 1.0 after periastron. A systematic velocity shift occurs in the apparent excess absorption, which moves from near $-60 \mathrm{~km} \mathrm{~s}^{-1}$ to near $+70 \mathrm{~km} \mathrm{~s}^{-1}$ within a timespan of 1 day. The narrow features that prevail near apastron cross the line profile on timescales $\sim 1.2$ day, although the timescale over which a complete set of bumps repeats the cycle is slightly longer, $\sim 1.8$ days.

The overall picture of the variability is best illustrated using a dynamic plot of line profile residuals. The residuals are obtained by subtracting from each perturbed line profile its corresponding unperturbed profile, for the same orbital phase and therefore, shifted by the same orbital motion. These residuals are then stacked one upon the other, separated vertically by the time interval between them ${ }^{1}$. A grey-scale representation is illustrated in Fig. 6, where we plot the data covering a complete orbital cycle plus 1.8 days, starting at periastron passage at the bottom of the figure. The separation in time between subsequent individual residuals is 0.02 day. The two patterns described above can now be clearly identified: broad bands with $\sim 1.7$-day spacing that are most prominent near periastron and closely-spaced narrow bands that dominate near apastron. The disruption of the narrow-band pattern by the approaching periastron suggests that the narrow features may be associated with the free oscillation modes. De Cat et al. (2000) note that the orbital eccentricity of the system could have a strong influence on the selection of the pulsation modes,

${ }^{1}$ We note that our residuals differ from those generally presented based on observational data, where the average profile over the observing run is used as the non-pulsation profile. Hao (1998) demonstrates that the average observed profile differs somewhat from the actual nonpulsation profile. 


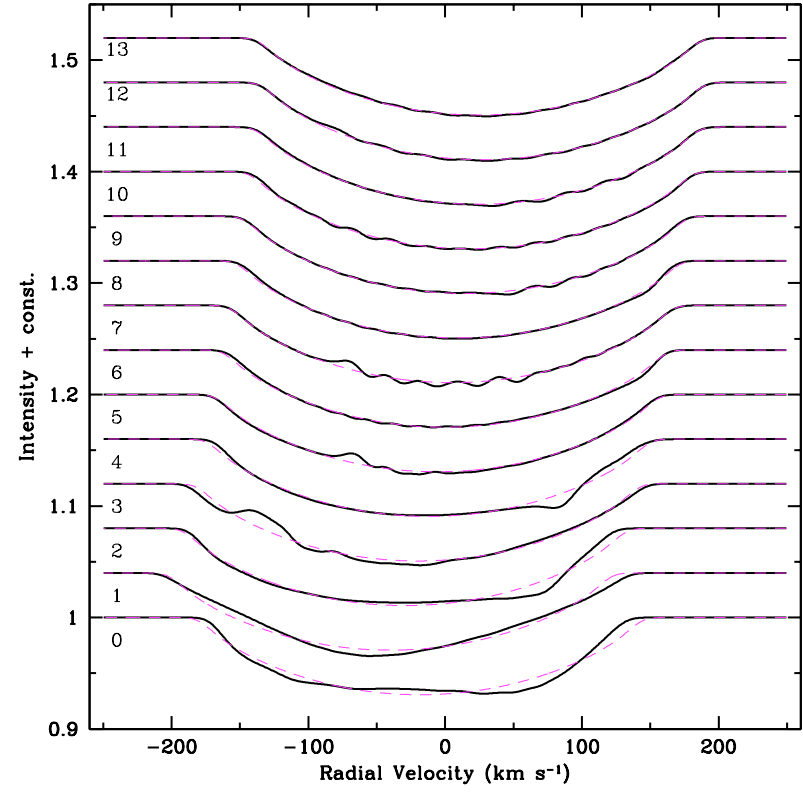

Fig. 4

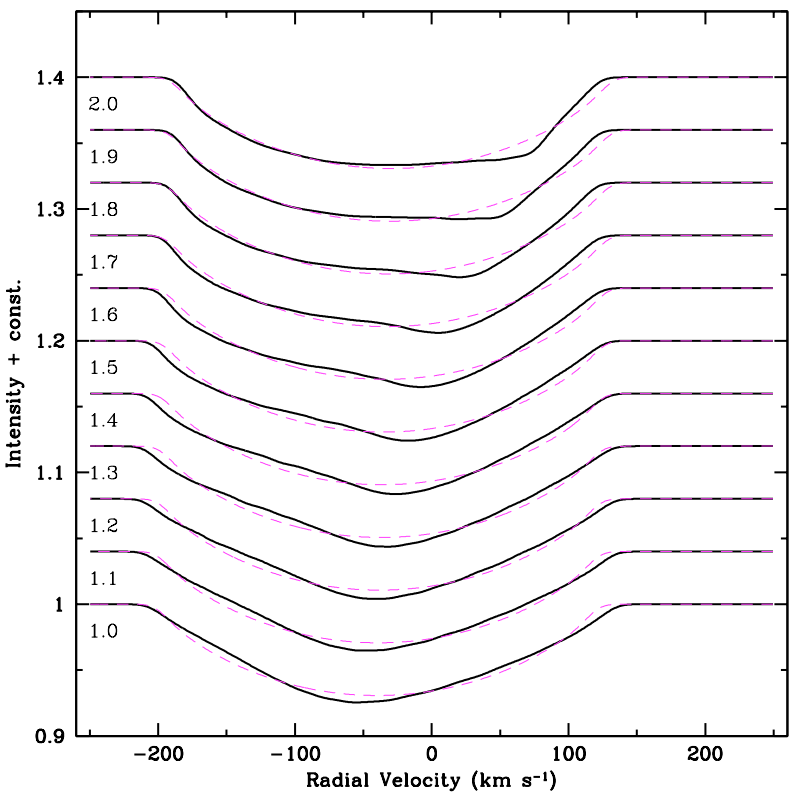

Fig. 5

Fig. 4. Sample of predicted line profiles for a model having parameters like those of $\epsilon$ Per, stacked with time increasing from bottom to top. Labels on the left indicate time (in days) after periastron. The dash lines are the corresponding rotationally-broadened absorption profiles, without the effects due to the oscillations. The velocity shifts of the line centroid correspond to the orbital motion.

Fig. 5. Sample of predicted line profiles for the same model as in the previous figure covering the timespan 1-2 days after periastron. The vertical shift is the same for all profiles. The dash line corresponds to the rotationally-broadened, unperturbed line profile. Note the apparent excess absorption near line center that crosses the perturbed line profile from blue to red within this timespan.

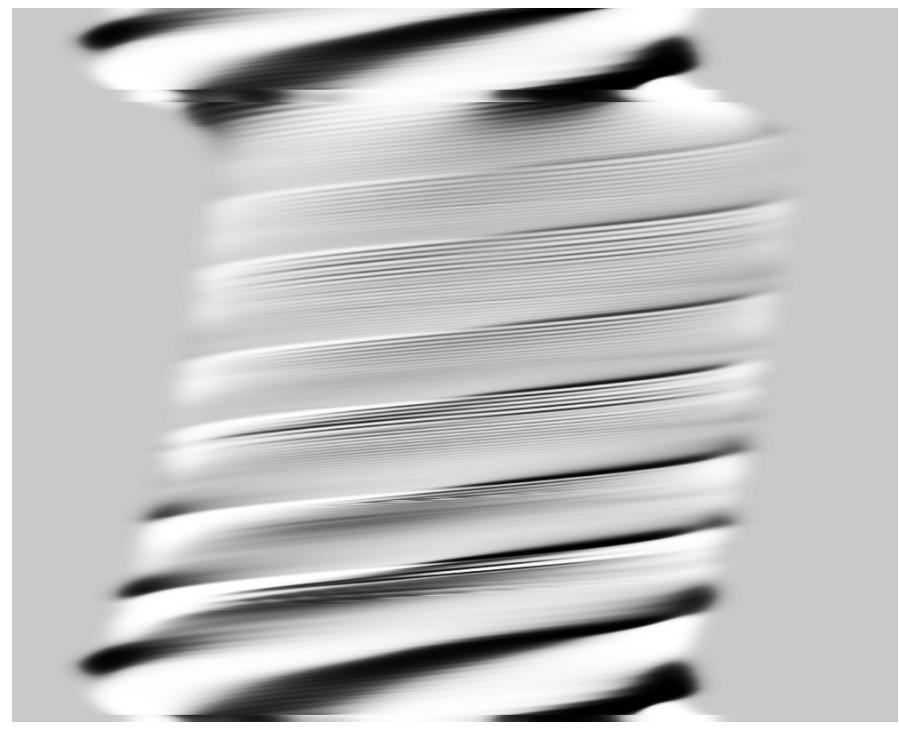

Fig. 6. Grey-scale plot of the differences between the perturbed line profile and the corresponding unperturbed profile, stacked in order of increasing time, starting at periastron passage (bottom) for a model having the parameters of $\epsilon$ Per. The top of the figure, which ends 1.8 days after the second periastron passage, illustrates the transition from the narrow-band pattern produced by multiple bumps on the line profiles to one in which fewer but stronger bumps prevail. Black represents the strongest absorption. The abscissa is velocity (in $\mathrm{km} \mathrm{s}^{-1}$ ), as in Figs. 4 and 5 . The radial velocity variations correspond to the orbital motion.

with forced oscillations occuring near periastron while free modes dominating near apastron, consistent with the results of our calculation.

The skewness (Press et al. 1992) is a useful parameter to quantify the effects of line-profile variability. A negative skewness means the presence of a blue tail; positive, a red tail. The bottom panel (left) of Fig. 7 illustrates the skewness variations as a function of time starting at periastron passage. The dash line corresponds to the unperturbed line profiles which, clearly, present 

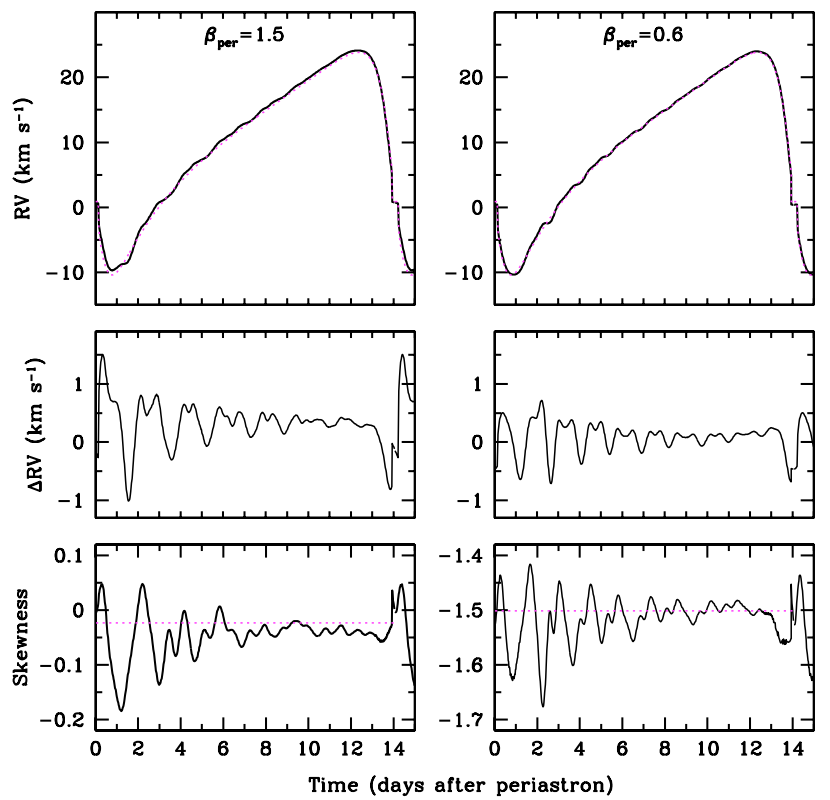

Fig. 7

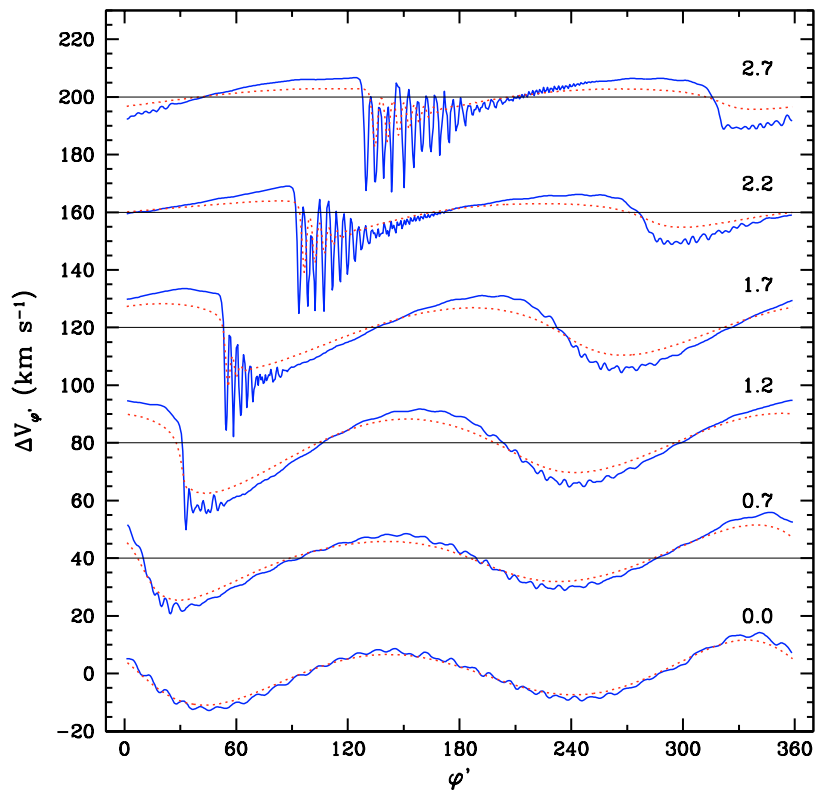

Fig. 8

Fig. 7. Radial velocity curves from the centroids of the perturbed and unperturbed (dotted) line profiles (top), difference between these centroids (middle), and skewness of the line profiles (bottom). The left panels correspond to a model with the $\epsilon$ Per parameters and the right panels correspond to the same set except for the rotation parameter, $\beta_{\text {per }}$, which in this case is sub-synchronous at periastron. In both cases, the perturbation produces a wobble in the RV curve and an oscillation in the skewness, particularly notable shortly after periastron passage.

Fig. 8. Azimuthal velocity difference between the external layer and the rigidly-rotating inner region as a function of azimuth angle along the equatorial belt $\left(\varphi^{\prime}=0\right.$ corresponds to the direction of the companion). Curves for different times during the orbital cycle are stacked, with time (labels in days after periastron) increasing towards the top of the figure. Vertical displacement is a constant $+40 \mathrm{~km} \mathrm{~s}^{-1}$ for each set of curves, for display purposes. Continuous and dotted lines correspond, respectively, to kinematic viscosity $v=0.002$ and $0.005 R_{\odot}^{2}$ day $^{-1}$. The larger kinematic viscosity reduces the oscillation amplitudes.

no variability. The skewness reflects the presence of the blue-to-red moving features. Its variation amplitudes are largest near periastron, decreasing after periastron passage.

Figure 7 (top left) displays the RV curves that are obtained by measuring the centroid of the perturbed (solid) and unperturbed (dash) line profiles. The values were shifted by $+10 \mathrm{~km} \mathrm{~s}^{-1}$ according to the reported systemic velocity of $\epsilon$ Per (De Cat et al. 2000). The dominant effect of the perturbations is to introduce a "wobble" in the RV curve about the curve that describes the orbital motion. The magnitude of the effect is illustrated in the middle panel of Fig. 7, where we plot the difference between the perturbed and the unperturbed RV curves. As expected, the "wobble" is largest at periastron, and decreases as apastron is approached. The cycles of the "wobble" have timescales in the range 1-2 days, and amplitudes in the range $\pm 1-1.5 \mathrm{~km} \mathrm{~s}^{-1}$. This range is actually smaller than the observed fluctuations in the RV curves (Gies et al. 1999; De Cat et al. 2000), which may be a consequence of our use of Gaussian profiles (see Sect. 4.4), combined with the use of a large value for the viscosity (see Sect. 4.2).

The right panel of Fig. 7 illustrates that the amplitude and frequency in the "wobble" depend on stellar rotation. In the example plotted in this panel, $\beta_{\mathrm{per}}=0.6$, and this leads to smaller amplitude $\left(<1 \mathrm{~km} \mathrm{~s}^{-1}\right)$ fluctuations.

\subsubsection{Retarding effect of gravitational potential on fluid motions}

The numerical model for predicting the line profile variability allows us to evaluate the relative contribution from each component of the oscillation amplitudes. We find that the radial component has negligible effects on the line profile. It is the tangential component that is responsible for nearly all the variability shown in the previous section. In Fig. 8 we plot the tangential component of the linear velocity perturbation, $\Delta V_{\varphi^{\prime}}=\left[\omega^{\prime}\left(\varphi^{\prime}\right)+|\Omega|-\left|\omega_{*}\right|\right] R_{1}$ as a function of $\varphi^{\prime} . \Delta V_{\varphi^{\prime}}$ is the difference between the azimuthal velocity at the stellar surface and the azimuthal velocity of the rigidly-rotating inner region. The continuous lines plot $\Delta V_{\varphi^{\prime}} \operatorname{cor}-$ responding to the line profile variability of $\epsilon$ Per described in the above section. At periastron, the curves are sinusoidal-like, with extrema at $\sim 140^{\circ}$ and $330^{\circ}\left(\sim+10 \mathrm{~km} \mathrm{~s}^{-1}\right)$, and at $\sim 40^{\circ}$ and $230^{\circ}\left(\sim-10 \mathrm{~km} \mathrm{~s}^{-1}\right)$. The surface elements located at $\sim 40^{\circ}$ have velocities slower than the rigid-body rotation because, after they have passed below the sub-stellar point and are rotating away from the companion, they are "held back" by the gravitational field of the companion. This "pull" leads to the appearance at this 

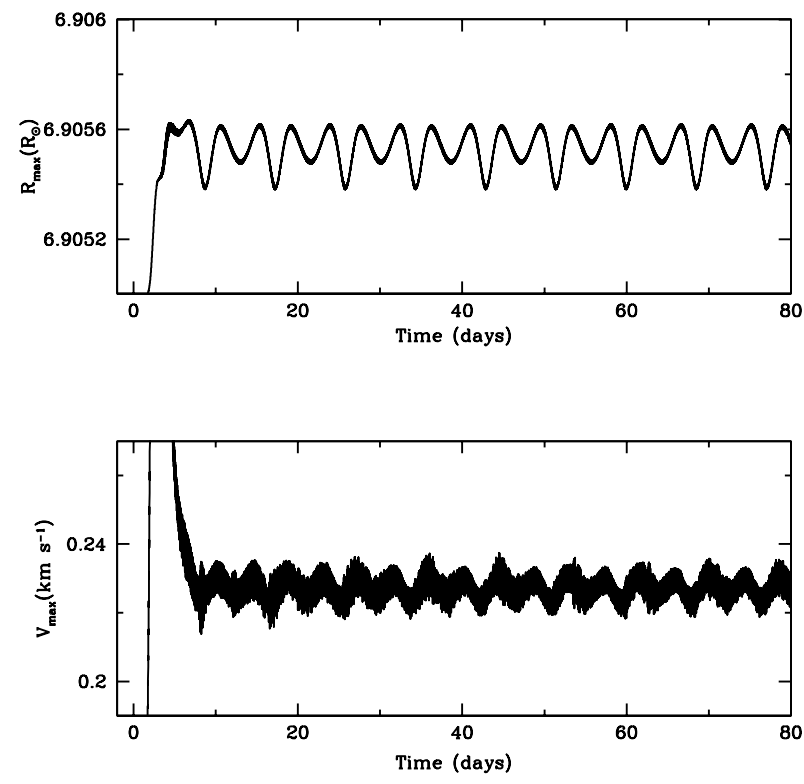

Fig. 9
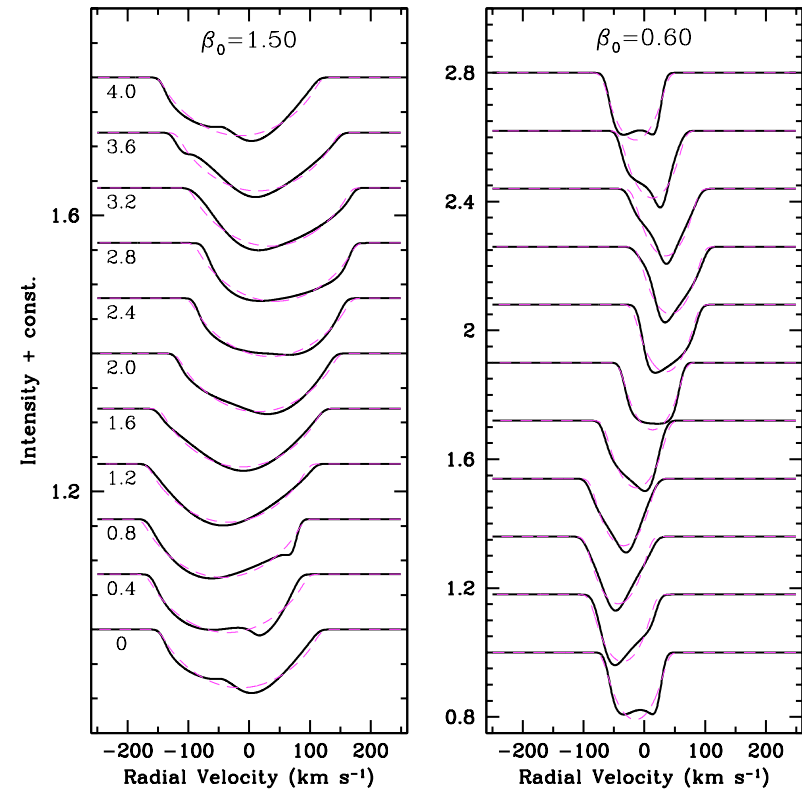

Fig. 10

Fig. 9. Temporal behavior of $R_{\max }$ and $V_{\max }$ for a short period $(P=4.03$ days), circular orbit binary system having the same masses and stellar radius as the $\epsilon$ Per system.

Fig. 10. Sample line profile variations for the circular non-synchronous orbit cases: super-synchronous rotation (left) and sub-synchronous rotation (right). Time increases upward. All parameters are the same in both cases except for the stellar rotational velocity.

location of large frequency oscillations that start to develop $\sim 1$ day after periastron. The amplitude of these oscillations increases significantly $\left(\sim-30 \mathrm{~km} \mathrm{~s}^{-1}\right)$, as the stellar rotation carries them to larger azimuth angles.

The dotted lines in Fig. 8 correspond to a calculation with a larger kinematic viscosity than used in the previous section, $v=0.005 R_{\odot}^{2}$ day $^{-1}$, showing that increasing the value of $v$ has the effect of decreasing the oscillation amplitudes.

In summary, one of the effects of the viscosity is to retard the advance of surface elements that have passed the sub-stellar point, and increase the velocity of those that are approaching the sub-stellar point. Because the internal regions of a star (regardless of what is assumed for its structure) must be rotating at a constant rotation rate on orbital timescales, Fig. 8 shows us that a surface differential rotation structure develops on the star at the locations along the equatorial belt where $\Delta V_{\varphi^{\prime}}$ differs from zero.

\subsection{Circular, non-syncronous orbit}

To illustrate the results obtained for circular, non-synchronous binary systems, we selected a short-period $\left(P_{\text {orb }}=4.03\right.$ days $)$ binary with the same $m_{1}, m_{2}, R_{1}$ and $\beta_{0}=\beta_{\text {per }}$ as in the eccentric case, but with a larger kinematic viscosity. Figure 9 illustrates the variations of $R_{\max }$ and $V_{\max }$. After the initial transitory phase of the calculation which lasts approximately two orbital cycles, $R_{\max }$ undergoes periodic oscillations. Note, however, that the period of these oscillations ( $\sim 9$ days) is longer than both the orbital period and the rotation period. We find such super-orbital periods for circular non-synchronous binary systems in general (Koenigsberger et al. 2003), and only for $\beta_{0}=0$ does the oscillation period equal the orbital period. However, given the weak dependence of the line profile variability predicted by our current model on the radial amplitudes of oscillation, these superperiods do not appear in the line profile variations.

The line profiles (Fig. 10) undergo strong changes. However, we do not find a large number of bumps as in the eccentric case near apastron. The dynamic plot of the difference between the perturbed and the unperturbed line profiles (Fig. 11) is characterized by broad bands of alternating excess absorption (dark) and excess emission (light) that cross the profile from the blue edge to the red, similar to the dynamic plot of the eccentric binary near periastron. Numerous narrow bumps that may be identified with the free oscillations are not evident.

The radial velocity curve, the difference between perturbed and unperturbed RV curves and skewness are plotted in Fig. 13 (top, middle, and bottom left, respectively). Instead of the numerous "wobbles" present in the eccentric orbit case, the difference between the perturbed and unperturbed RV curves displays only two maxima and minima. Similarly, the skewness follows a sinusoidal curve with two unequal maxima and minima per orbital cycle.

Figure 12 and the right-hand panels of Fig. 13 illustrate the grey-scale plot and the variability characteristics of a binary in which $m_{1}$ is rotating at a sub-synchronous speed $\left(\beta_{0}=0.60\right)$, and the corresponding sample line profiles are in the right panel of 


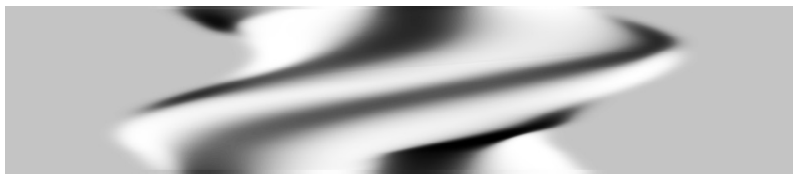

Fig. 11

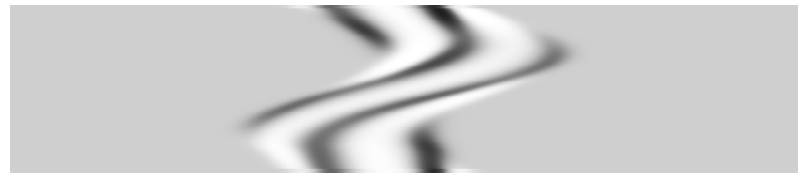

Fig. 12

Fig. 11. Grey-scale plot of the line-profile differences for the $\beta_{0}=1.50$ circular orbit binary system. One orbital cycle is illustrated, with time starting at the bottom of the image. Only the broad-band patterns are apparent.

Fig. 12. Grey-scale plot of the line-profile differences, as in Fig. 11, but for $\beta_{0}=0.60$.
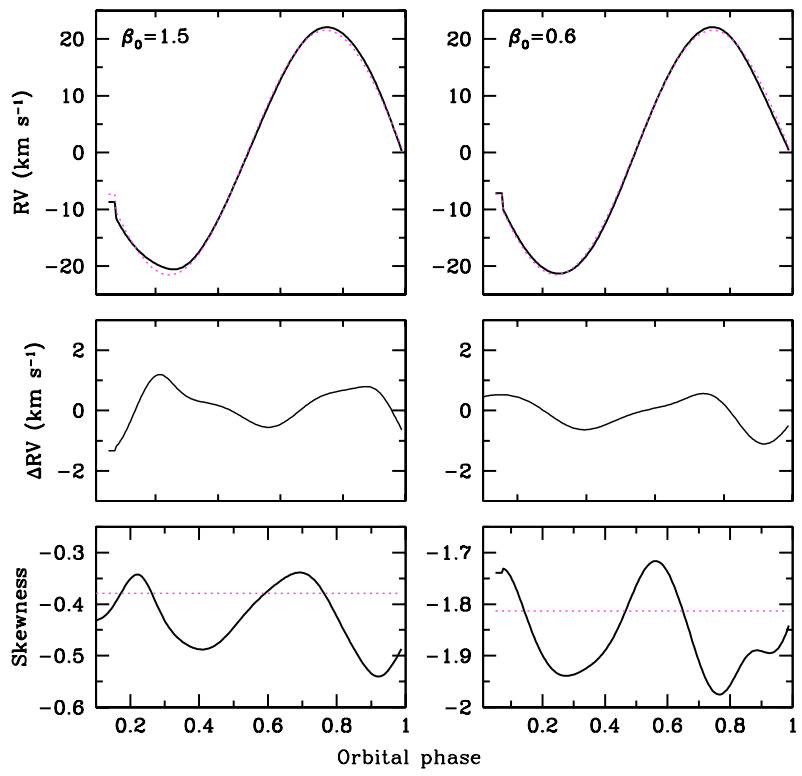

Fig. 13
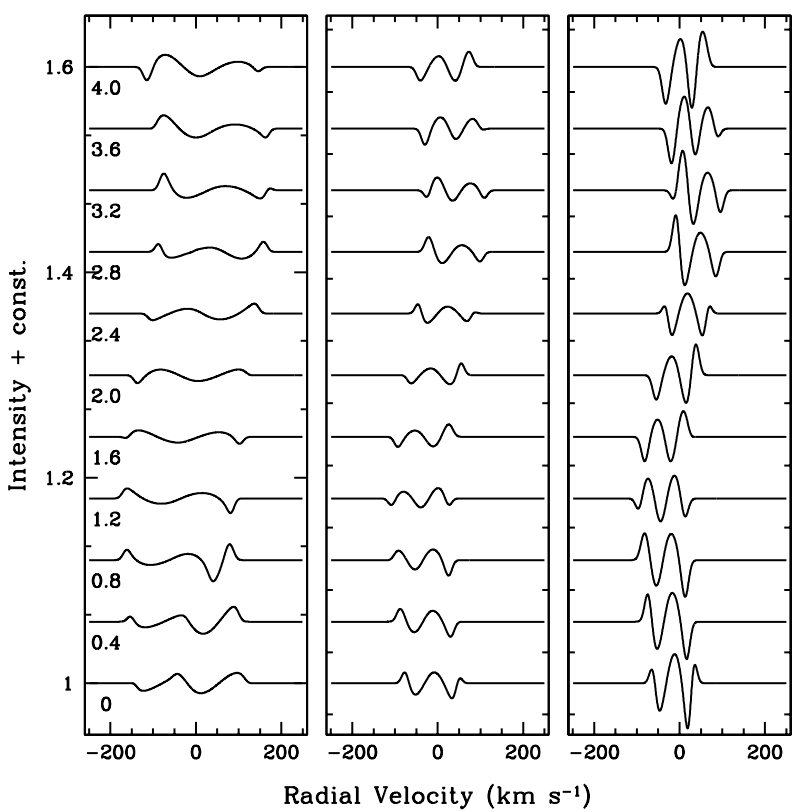

Fig. 14

Fig. 13. Radial velocity curves (top), difference between perturbed and unperturbed RV curves (middle), and the skewness of the line profile (bottom) for two circular orbit cases: super-synchronous (left), and sub-synchronous (right). Dotted lines correspond to the unperturbed line profiles.

Fig. 14. Difference between the perturbed and the corresponding unperturbed line profiles for the circular orbit case, for three different values of $\beta_{0}: 1.50$ (left), 0.77 (middle) and 0.60 (right). Time increases from bottom to top, the labels indicating time (in days) after the initial profile are the same as in Fig. 10.

Fig. 10. Although the general characteristics of the RV curve and the skewness are the same as in the super-synchronous case, the detailed behavior differs, particularly, the location of the maxima and minima.

The two cases we present above, $\beta_{0}=1.50$ and 0.60 , correspond, respectively, to the $3: 2$ and 3:5 resonances between the stellar rotation and orbital angular velocities. We find that the inverted "S" pattern in the grey-scale representation of the variability, and the overall behavior of the line profiles shown in the Figs. 10-13 are typical of the predictions for the circular, non-synchronous short-period systems in general. In Fig. 14 we illustrate a sample of the line-profile residuals for the two cases already discussed, and for $\beta_{0}=0.77$ (middle panel), showing that the amplitudes and qualitative behavior for $\beta_{0}=0.77$ are not significantly different from those of $\beta_{0}=1.50$; the largest amplitudes are obtained for $\beta_{0}=0.60$. A more in-depth study of the dependence of the line profile variability on $\beta_{0}$, the viscosity and the binary parameters is left for a forthcoming investigation.

As with the eccentric case, the dominant cause of line-profile variability in the circular orbit is the azimuthal component of the velocity. A clear difference, however, is that the azimuthal velocity structure in the frame of reference that rotates with the orbital motion of $m_{2}$ remains constant throughout the orbital cycle. This is because the orbital separation is constant at all times. Figure 14 illustrates this velocity structure for the three circular orbit cases, $\beta_{0}=1.50,0.77$ and 0.60 , showing that the amplitudes and the location of maxima and minima depend on the value of $\beta_{0}$.

\section{Conclusions}

We present the results of ab initio calculations of the photospheric line-profile variability expected from the time-variable tidal deformations in binary systems. We use the one-layer approximation to compute the effects produced on a primary star's 


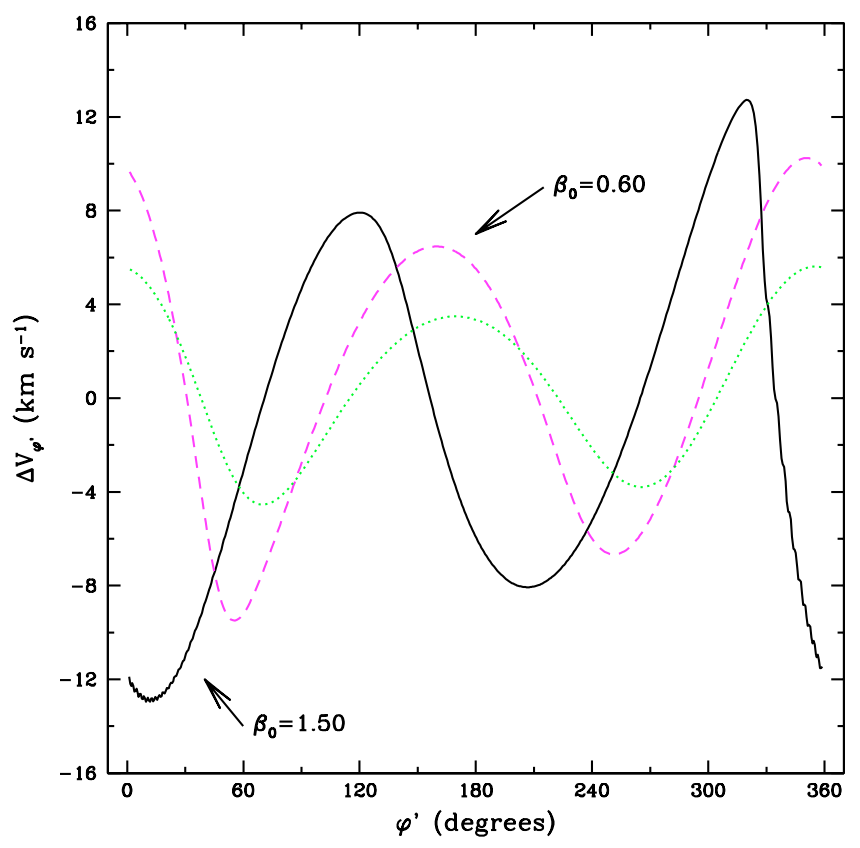

Fig. 15. Tangential velocity difference between the external layer and the rigidly-rotating inner region, as a function of azimuth angle along the equatorial belt of $m_{1}$ for circular orbits with $\beta_{0}=1.50$ (continuous line), 0.60 (broken line) and 0.77 (dotted line). Contrary to what occurs in an eccentric orbit (Fig. 8), the angular distribution in the corotating reference frame does not change with orbital phase.

equatorial region by its companion in the general case of arbitrary equatorial rotational velocity $\left(V_{\text {eq }}\right)$, eccentricity and viscosity, $v$. No assumptions regarding the modes of oscillation are made. Although this model is very simplified, it reproduces many of the features that are observed in the line profile variability of $\beta$ Cepheid binary systems, particularly the presence and multiplicity of blue-to-red moving bumps. For an eccentric binary with parameters like those of the B0.5 IV star $\epsilon$ Per, the timescale over which the pattern of bumps is predicted to cross the rotationally broadened line profile is $\sim 1-2$ days. This is longer than the reported timescales of hours from the observation (Gies \& Kullavanijaya 1988; Gies et al. 1999; De Cat et al. 2000).

The line profiles display the strongest variability around periastron, reflecting the fact that the forced oscillations dominate during these orbital phases. The number and intensity of the bumps change with orbital phase: fewer but stronger bumps are present near periastron, while close to a dozen weaker bumps are predicted near apastron.

In circular non-synchronous orbits, the bumps are few in number and broad, resembling those that are predicted near periastron in the eccentric case described above. A grey-scale representation of the variability displays excess absorption which moves in an inverted " $S$ " pattern for both the super-synchronous and sub-synchronous cases we tested. No line profile variability is predicted for the equilibrium case $\left(\beta_{0}=1.00\right)$.

These results show that even though our model is restricted to the calculation of the effects on a single surface layer of the star and only in the equatorial belt, significant insight can be gained into the role played by the various parameters in producing line-profile variability. In particular, it provides a tool for determining the type of variability that is expected from the forced oscillations, and differentiating it from the variability produced by the free oscillation modes. In addition, the results of our calculations raise interesting questions in a wider context, which we summarize below.

1. Deviations from the true radial velocity curves: in both the eccentric and the circular orbit cases, the variability of the line profiles leads to deviations of the radial velocity curve one would derive from the measurement of the centroid of the photospheric absorption, with respect to the actual radial velocity curve. For the examples given in this paper, the deviations are $<2 \mathrm{~km} \mathrm{~s}^{-1}$, but larger values are expected for systems in which the secondary component is more massive than the $m_{2}=2 M_{\odot}$ that we have adopted for our present calculations. This effect impacts directly the determination of the stellar masses from the solution of radial velocity curves and may introduce a significant uncertainty in the determination of the masses of unseen companions.

2. Constraints on the numerical value of the viscosity: the line-profile variability computed by our model is caused primarily by the azimuthal component of the velocity perturbations, $\Delta V_{\varphi^{\prime}}$, accross the stellar surface. For a given set of stellar and orbital parameters, the magnitude of $\Delta V_{\varphi^{\prime}}$ depends on the value of $v$, the kinematic viscosity, which is a free parameter of the code. The possibility thus arises of constraining the values of $v$ near the stellar surface through the comparison of theoretical and observed line-profile variability. For example, we find $v<1.1 \times 10^{14} \mathrm{~cm}^{2} \mathrm{~s}^{-1}$ for the parameters of $\epsilon$ Per which, assuming a temperature of $T \sim 30000 \mathrm{~K}$, corresponds to a Shakura \& Sunyaev (1973) parameter $\alpha<0.001$. This type of result is relevant to studies involving the structure of accretion disks. 
3. Differential rotation in surface layers and magnetic activity: because the shear produced by the relative azimuthal motions of different stellar layers can lead to magnetic field generation (see, for example, Spruit 2002; and Blackman 2000, and references therein), we put forth the hypothesis that stars in non-synchronous binary systems may have significantly more surface activity than their single-star or synchronously-rotating binary counterparts. The manifestation of this activity is likely to be the presence of emission lines and/or enhanced X-ray luminosity. For example, the X-ray luminosity characteristics of the sample of B8-9 V binaries studied by Haro et al. (2004) may be associated with this phenomenon. It is also tempting to suggest that magnetic activity located close the the equatorial plane in the binary RY Per may be responsible for the highly non-spherical distribution of extended $\mathrm{H} \alpha$ emission that was resolved by Barai et al. (2004). Clearly, the timescale that is needed for seed poloidal magnetic field lines to be wrapped into a toroidal configuration may be significantly longer than the large amplitude oscillations in $\Delta V_{\varphi^{\prime}}$, which occur on orbital timescales. However, the net effect of the tidal interaction in binaries is to drive the system towards corotation and, as shown by Goldreich \& Nicholson (1989), tidal despinning to synchronous rotation procedes from the outside toward the inside of the star. Hence, very young binaries, or binaries that have undergone significant mass-loss could be in the early stages of becoming synchronous; that is, the outer layers may rotate nearly synchornously, while the interior is still far from corotation. Hence, a dynamo mechanism could be in effect very close to the surface of the star. It is interesting to note that this mechanism could account for the presence of strong magnetic fields even on the surface of single massive stars in which strong mass-loss through stellar winds or LBV-type ejections have removed angular momentum from external layers.

4. Peculiar mass-shedding events: the possibility of a connection between stellar pulsations and mass loss has been suggested for a wide variety of stars, ranging from Be-type stars (cf., Smith \& Ebbets 1981; Vogt \& Penrod 1983; Lee 1993; Rivinius et al. 1998; Gies et al. 1999), cool hypergiants (cf. de Jager et al. 1997), Miras (Willson 2000, and references therin), and erupting Wolf-Rayet type binaries (Koenigsberger et al. 2002), among others. However, the precise mechanism for inducing significant mass-loss through pulsations has been elusive. It is interesting to note that azimuthal speeds of $\sim 13 \mathrm{~km} \mathrm{~s}^{-1}$, as we find in our calculations, imply that a large quantity of energy may be transferred from the orbital motion of the binary companion to internal thermal energy, through the action of the viscous forces. Thus, it may prove very interesting to analyze the energy deposition and transport mechanism in a scenario where a fraction of the orbital energy may be channeled to drive mass-loss.

Acknowledgements. We would like to thank Petr Harmanec for encouraging us to pursue the line-profile calculation, and Myron Smith for a critical reading of the manuscript and very valuable comments. G.K. thanks Norbert Langer, Doug Gies, Susana Lizano and Moira Jardine for enlightening discussions, and the Instituto de Astronomía of UNAM for computing support. We thank the referee for the valuable suggestions. This work was supported by CONACYT grant 36569E and UNAM/DGAPA grant IN118202.

\section{References}

Barai, P., Gies, D. R., Choi, E., et al. 2004, ApJ, 608, 989

Blackman, E. G. 2000, ApJ, 529, 138

Bolton, C. T. 1983, Hvar Obs. Bull., 7, 241

Briquet, M., Aerts, C., \& De Cat, P. 2001, A\&A, 343, 872

Carroll, J. A. 1928, MNRAS, 88, 548

Chandrasekhar, S. 1933, MNRAS, 93, 463

Chandrasekhar, S. 1957, An Introduction to the Study of Stellar Structure (New York: Dover publications, Inc.)

De Cat, P., Telting, J., Aerts, C., \& Mathias, P. 2000, A\&A, 359, 539

de Jager, C., Lobel, A., \& Israelian, G. 1997, A\&A, 325, 714

De Ridder, J., Dupret, M.-A., Neuforge, C., \& Aerts, C. 2002, A\&A, 385, 572

Fehlberg, E. 1968, NASA TR R-287

Gies, D. R., \& Kullavanijaya, A. 1988, ApJ, 326, 813

Gies, D. R., et al. 1999, ApJ, 525, 420

Goldreich, P., \& Nicholson, P. D. 1989, ApJ, 342, 1079

Harmanec, P. 1989, Bull. Astron. Inst. Czechoslovakia, 40, 201

Harmanec, P. 1999, A\&A, 341, 867.

Harmanec, P., \& Tarasov, A. E. 1990, Bull. Astron. Inst. Czechoslovakia, 41, 273

Hao, J. 1998, ApJ, 500, 440

Haro, S., Juárez, J. A., \& Koenigsberger, G. 2004, in Stellar Rotation, ed. A. Maeder, \& P. Eenens (San Francisco: ASP-CS), Proc. IAU Symp., 215,163

Hubeny, I., Heap, S. R., \& Altner, B. 1991, ApJ, 377, L33

Hut, P. 1980, A\&A, 92, 167

Hut, P. 1981, A\&A, 99, 126

Kiriakidis, M., El Eid, M. F., \& Glatzel, W. 1992, MNRAS, 255, 1

Koenigsberger, G., Moreno, E., \& Cervantes, F. 2002, in Interacting Winds from Massive Stars, Proc. Int. Workshop, Les Iles-de-la-Madeleine, Quebec, Canada, ed. A. F. J. Moffat, \& N. St.-Louis, ASP Conf. Ser., 260, 507 
Koenigsberger, G., Moreno, E., \& Cervantes, F. 2003, in A Massive Star Odyssey, from Main Sequence to Sueprnova, ed. K. A. van der Hucht, A. Herrero, \& C. Esteban, Proc. IAU Symp., 212, 101

Kumar, P., Ao, C. O., \& Quataert, E. J. 1995, ApJ, 449, 294

Landau, L. D., \& Lifshitz, E. M. 1984, Fluid Mechanics (Pergamon Press)

Lee, U. 1993, ApJ, 417, 697

Lee, U., \& Saio, H. 1990, ApJ, 349, 570

Lennon, D. J., Kudritzki, R. P., Becker, S. R., et al. 1991, A\&A, 252, 498

Moreno, E., \& Koenigsberger, G. 1999, RMA\&A, 35, 157

Moskalik, P., \& Dziembowski, W. A. 1992, A\&A, 256, L5

Osaki, Y. 1971, PASJ, 23, 485

Press, W. H., \& Teukolsky, S. A. 1977, ApJ, 213, 183

Press, W. H., Teukolsky, S. A., Vetterling, W. T., \& Flannery, B. P. 1992, Numerical Recipes for C Programming (New York: Cambridge University Press)

Rivinius, Th., Baade, D., Stefl, S., et al. 1998, in A Half Century of Stellar Pulsation Interpretations, ed. P. A. Bradley, \& J. A. Guzik, ASP Conf. Ser., 135, 343

Rocca, A. 1987, A\&A, 175, 81

Savonije, G. J., Papaloizou, J. C. B., \& Alberts, F. 1995, MNRAS, 277, 471

Saio, H., Kambe, E., \& Lee, U. 2000, ApJ, 543, 359

Shakura, N. I., \& Sunyaev, R. A. 1973, A\&A, 24, 337

Smeyers, P., Willems, B., \& Ban Hoolst, T. 1998, A\&A, 335, 622

Smith, M. A. 1977, ApJ, 215, 574

Smith, M. A. 1985a, ApJ, 297, 224

Smith, M. A. 1985b, ApJ, 297, 206

Smith, M. S., \& Ebbets, D. 1981, ApJ, 247, 158

Smith, M. A., \& Buta, R. J. 1979, ApJ, 232, L193

Smith, M. A., Fullerton, A. W., \& Percy, J. R. 1987, ApJ, 320, 768

Spruit, H. C. 2002, A\&A, 381, 923

Symon, K. R. 1971, Mechanics (Addison-Wesley)

Tarasov, A. E., Harmanac, P., Horn, J., et al. 1995, A\&AS, 110, 59

Telting, J. H., \& Schrijvers, C. 1997, A\&A, 317, 723

Townsend, R. H. D. 1997, MNRAS, 284, 839

Vogt, S. S., \& Penrod, G. D. 1983, ApJ, 275, 661

Willems, B. 2003, MNRAS, 346, 968

Willems, B., \& Aerts, C. 2002, A\&A, 384, 441

Willson, L. A. 2000, ARAA, 28, 573

Witte, M. G., \& Savonije, G. J. 1999, A\&A, 350, 129

Witte, M. G., \& Savonije, G. J. 2001, A\&A, 366, 840

Zahn, J.-P. 1970, A\&A, 4, 452

Zahn, J.-P. 1977, A\&A, 57, 383

Zahn, J.-P. 1989, A\&A, 220, 112 